\title{
Adicción al ejercicio medida a través del Exercise Addiction Inventory (EAI) y salud en deportistas habituales. Una revisión sistemática y meta-análisis Exercise addiction measure through the Exercise Addiction Inventory (EAI) and health in habitual exercisers. A systematic review and meta-analysis
}

\author{
Javier Simón-Grima*, Nerea Estrada-Marcén*, Jesús Montero-Marín**. \\ * Facultad de Ciencias de la Salud y del Deporte. Universidad de Zaragoza, Huesca, Spain. \\ ** Red de Investigación en Atención Primaria (REDIAPP), Zaragoza, Spain.
}

\section{Resumen}

Cada vez es más frecuente la investigación sobre adicción al ejercicio debido a la importancia del exceso de actividad física en la salud general. Diferentes estudios han investigado la prevalencia del riesgo de adicción al ejercicio (RAE) y sus consecuencias, existiendo contradicciones con respecto a la asociación entre el RAE y las variables asociadas al entrenamiento. Uno de los objetivos de esta revisión sistemática fue analizar los estudios que han usado el Exercise Addiction Inventory (EAI) para valorar el RAE, así como realizar un meta-análisis para observar las posibles diferencias entre grupos con y sin RAE respecto a la prevalencia, edad, variables de salud (calidad de vida física y mental, desórdenes alimentarios) o de entrenamiento físico (horas/semana). Las búsquedas de estudios se realizaron en bases de datos electrónicas como Pubmed, SPORTDiscus o Scopus, y se hicieron utilizando términos de indexación y palabras clave relacionados con materias médicas o ciencias del deporte. Los criterios de inclusión fueron: participantes evaluados con el EAI; resultados reportados de prevalencia de RAE y/o puntuación en dicho cuestionario; diseño observacional. Veinte estudios cumplieron los criterios de elegibilidad para la revisión sistemática y 17 fueron incluidos en el meta-análisis. Los resultados mostraron peores valores en calidad de vida mental y trastornos de alimentación en el grupo con RAE comparado con el grupo sin RAE. Además, el grupo con RAE era más joven y realizaba más horas de entrenamiento semanal. No obstante, es necesaria más investigación.

Palabras clave: Adicción; Trastorno; Actividad física; Calidad de vida; Entrenamiento.

\begin{abstract}
Research on physical exercise addiction is becoming more frequent due to the importance of excessive physical activity on health in general. Different studies have investigated the prevalence of risk of exercise addiction (REA) and its consequences. Furthermore, there exist a series of contradictions regarding the relationship between REA and other variables associated with physical training. One goal of this systematic review and meta-analysis consists of analysing possible differences in prevalence, age, general health (mental and physical quality of life, eating disorders) and physical training (hours/week) between groups with REA and non-addicted groups. The Exercise Addiction Inventory (EAI) was used to undertake this research. Research was carried out in electronic databases such as Pubmed, SPORTDiscus or Scopus. Inclusion criteria: Studies were eligible as long as participants were measured with EAI, results showed prevalence of REA and/or EAI score, and the study was observational. Twenty studies met the established eligibility criteria for inclusion in the systematic review, whereas seventeen studies were included in the meta-analysis. Regarding mental quality of life, results showed lower values for the exercise addiction risk group, compared with the non-addicted group. The exercise addiction group was younger than the non-addicted group and dedicated more weekly hours to physical training. Subjects with REA have a lower health profile than those with non-REA. However, more research is required, given the lack of consensus on how to measure exercise addiction and the scarce number of studies to date.

Keywords: Illegal drug use; Violence; Crime; Gender; Victim-offender overlap.
\end{abstract}

Recibido: Junio 2017. Aceptado: Febrero 2018.

Enviar correspondencia a: Javier Simón Grima. C/Julian Sanz Ibañez, nº42, Esc. 2, 4-C. 50017 Zaragoza, España

Teléfono 675323 197. E-mail: jsimongrima@hotmail.com 
A unque los beneficios del ejercicio son habitualmente bien conocidos (Kokkinos y Myers, 2010), la literatura creciente muestra la posibilidad que una minoría pequeña de personas experimente diversas consecuencias negativas del ejercicio en exceso (Berczik et al., 2012). Este motivo ha fundamentado la sugerencia que puede haber un límite al volumen de ejercicio antes que resulte en efectos negativos sobre la salud, como el riesgo de adicción al ejercicio (Szabo, Griffiths, de La Vega Marcos, Mervó y Demetrovics, 2015). La adicción al ejercicio se ha descrito como un patrón comportamental mórbido en el cual el individuo que hace ejercicio pierde el control sobre sus hábitos y actúa de forma compulsiva, muestra dependencia y experimenta resultados negativos sobre su salud y su vida profesional y social (Szabo et al., 2015). En este meta-análisis, el término "adicción" se consideró el más adecuado, dado el énfasis de algunos investigadores (Berczik et al., 2012) en el mismo al incluir la dependencia y la compulsión (Goodman, 1990). Una limitación importante es el uso de distintos términos para describir el mismo fenómeno, como "adicción el ejercicio" (Berczik et al., 2012), "dependencia del ejercicio" (Hausenblas y Downs, 2002a; Pasman y Thompson, 1988), "ejercicio obligatorio" (Pasman y Thompson, 1988), "abuso del ejercicio" (Davis, 2000) y "ejercicio compulsivo" (Dalle Grave, Calugi y Marchesini, 2008). Varios instrumentos se han desarrollado y revisado para valorar los efectos negativos del ejercicio excesivo (Allegre, Souville, Therme y Griffiths, 2009), como son el Compulsive Exercise Test (Taranis, Touyz y Meyer, 2011), el Exercise Dependence Scale (Hausenblas y Downs, 2002a), el Exercise Dependence Questionnaire (Ogden, Veale, y Summers, 1997) y el Exercise Addiction Inventory (EAI) (Griffiths, Szabo, y Terry, 2005), que ofrecen un rango de puntuaciones de riesgo en lugar de un diagnóstico (Szabo et al., 2015). Aunque las personas adictas al ejercicio desarrollan una pérdida de control de tal manera que el ejercicio se convierte en una obligación y en un exceso (Szabo et al., 2015; Mónok et al., 2012), la adicción al ejercicio no ha sido clasificada como un trastorno mental en la edición más reciente (quinta) del Diagnostic and Statistical Manual of Mental Disorders (DSM-5) (American Psychiatric Association, 2013).

Basado en los síntomas derivados de la adicción al ejercicio (Szabo et al., 2015), se desarrolló el EAI como instrumento de cribado para esta patología (Griffiths et al., 2005). Se compone de 6 preguntas en base a los 6 componentes generales de adicción (prominencia, cambio de estado de ánimo, tolerancia, síntomas de abstinencia, conflicto social, y recaída) (Griffiths, 1996). Las respuestas se puntúan en una escala de 5-puntos tipo Likert, desde 1 (totalmente en desacuerdo) a 5 (totalmente de acuerdo) y se calcula una puntuación global (entre 6-30 puntos), donde una puntuación de $\geq$ 24 indica la probabilidad de adicción al ejercicio. Diferentes estudios han mostrado resultados satisfactorios en cuanto a las propiedades psicométricas del EAI, con valores adecua- dos de validez y fiabilidad (Lichtenstein, Christiansen, Bilenberg, y Stoving, 2014; Lichtenstein y Jensen, 2016; Mónok et al., 2012; Sicilia, Alias-Garcia, Ferriz, y Moreno-Murcia, 2013). Además, una reevaluación intercultural reciente del EAI en cinco países mostró que sigue siendo un instrumento adecuado para evaluar la adicción al ejercicio, a pesar de algunas diferencias entre países (Griffiths et al., 2015).

Varios estudios han usado el EAI para investigar la adicción al ejercicio en diferentes muestras poblacionales y la prevalencia del riesgo de adicción al ejercicio y/o la relación entre el mismo y distintas variables de salud, tanto física como mental (Cunningham, Pearman, y Brewerton, 2016; De la Vega, Parastatidou, Ruiz-Barquin y Szabo, 2016; Li, Nie y Ren, 2015; Lichtenstein, Andries, Hansen, Frystyk y Stoving, 2015; Lichtenstein, Christiansen, Elklit, Bilenberg y Stoving, 2014; Lichtenstein y Jensen, 2016; Maraz, Urban, Griffiths y Demetrovics, 2015; Mayolas-Pi et al., 2017; Mónok et al., 2012; Sicilia et al., 2013; Szabo, De la Vega, Ruiz-Barquín y Rivera, 2013; Weinstein, Maayan y Weinstein, 2015). De todas maneras, existe una gran variabilidad entre los estudios sobre la prevalencia de RAE (Szabo et al., 2015). Además, la cantidad de exceso de ejercicio físico se ha destacado entre los muchos factores objetivos y subjetivos que pueden explicar dicha variabilidad (Adams, Miller y Kraus, 2003; Chapman y De Castro, 1990). En la actualidad, la relación entre el RAE y la cantidad de ejercicio físico es contradictoria (Cook et al., 2013; Szabo et al., 2013), dado que varios autores afirman que el ejercicio excesivo no es necesariamente indicativo de un trastorno, y que este hecho no es necesariamente inadaptado (Szabo et al., 2015). La relación entre el RAE y otras variables asociadas con el ejercicio, como frecuencia semanal, horas semanales, y otras, también es confusa o desconocida (Freimuth, Moniz, y Kim, 2011; Youngman y Simpson, 2014). Otra revisión sistemática realizada por Hausenblas y Downs (Hausenblas y Downs, 2002b) sobre la dependencia del ejercicio concluyó que los resultados eran inconclusos, en parte por la escasez de estudios experimentales, la inconsistencia o inexistencia de grupos de control, la discrepancia de los criterios operativos, o lo inapropiado de las medidas usadas de dependencia del ejercicio. Por lo tanto, y dado el aumento de estudios sobre la adicción al ejercicio en los últimos años (Szabo et al., 2015), es necesaria una revisión de meta-análisis para aclarar y unificar estos resultados para avanzar el conocimiento en este campo, evaluar las limitaciones metodológicas y ofrecer una visión global de la adicción al ejercicio definida por la puntuación en el EAI. Esto puede ser un punto de partida para revisiones sistemáticas futuras que incluyan más instrumentos para valorar dicho trastorno.

Por lo tanto, en personas que hacían ejercicio de forma habitual el objetivo principal de este estudio fue:

- Evaluar la posible existencia de diferencias en calidad de vida (física y mental) entre personas con RAE y personas sin RAE. 
Un objetivo secundario fue, en personas que hacían ejercicio de forma habitual fue:

- Valorar posibles diferencias en trastornos de alimentación, edad, y horas de entrenamiento físico por semana entre personas con y sin RAE.

\section{Método}

Esta revisión se realizó en línea con el PRISMA (Preferred Reporting Items for Systematic Reviews and Meta-Analyses) statement (Moher et al., 2015). Dicha revisión fue registrada en la base de datos internacional PROSPERO (International Database of Prospectively Systematic Reviews) con número de registro: CRD42017065252.

\section{Criterios de inclusión y exclusión}

Los estudios se incluyeron en esta revisión sistemática cuando:

- La adicción al ejercicio fue valorada con el EAI.

- Los estudios mostraron resultados sobre la prevalencia de la adicción al ejercicio y/o la puntuación en el cuestionario EAI.

- El estudio tenía un diseño observacional.

Excluimos aquellos estudios que únicamente valoraron la adicción al ejercicio con cuestionarios diferentes al EAI debido a la dispersión conceptual actual en este campo, y porque el EAI es el instrumento recomendado para valorar la adicción al ejercicio en general (Griffiths et al., 2015). Los estudios realizados en idiomas distintos el inglés y el español fueron excluidos. Además de los criterios ya mencionados, otros criterios de inclusión para este meta-análisis fueron:

El estudio informó de la prevalencia de REA y/o los resultados en calidad de vida física y mental, edad o trastornos de alimentación entre grupos con y sin RAE.

\section{Fuentes de información y estrategia de búsqueda}

Realizamos búsquedas iniciales en bases de datos electrónicas hasta el 27 de marzo del 2017, usando Pubmed, SPORTDiscus, Scopus, Science Direct y ProQuest Psychology Database. Actualizamos dicha búsqueda electrónica el 30 de mayo del 2017, usando Encabezados de Temas Médi$\cos (\mathrm{MeSH})$, términos de indexación de bases de datos, palabras clave y operadores booleanos $(\mathrm{Y} / \mathrm{O})$ en la estrategia de búsqueda. Los términos fueron agrupados por temas relacionados con adicción al ejercicio, dependencia del ejercicio y salud. Para Pubmed, los términos de búsqueda incluyeron: "exercise addiction" [Title] OR "dependence of exercise" [Title] AND EAI [Title/Abstract] OR "Exercise Addiction Inventory [Title/Abstract]. Para SPORTDiscus, usamos limitadores como English y Spanish; Journal Academical; TI (Title) "Exercise addiction" OR "dependence of exercise" AND AB (Abstract) "EAI" OR AB (Abstract) "Exercise Addiction Inventory". El mismo autor (JS) dirigió las búsquedas. Los resultados fueron comparados con el software Endnote (Thomson Reuters, New York), y los duplicados fueron eliminados. Revisamos el título y el resumen de los estudios restantes para detectar los relevantes (JS). Los textos íntegros de los estudios posiblemente adecuados fueron leídos por dos autores (JS/NE) para valorar su elegibilidad según los criterios de inclusión. Examinamos los listados de referencias de los manuscritos elegidos y de artículos de revisión relevantes para buscar estudios potencialmente elegibles (JS).

\section{Proceso de extracción de datos}

Extrajimos de los estudios seleccionados (JS) las características de estudio, que incluyeron el tamaño muestral, edad, peso, altura, nivel de estudios, estado civil, características de las muestras, tipo de ejercicio, medidas, grupos, puntuación EAI y/o prevalencia de adicción al ejercicio (AE), variables analizados (riesgo de lesión, RAE, nivel de actividad, calidad de vida física y mental, trastornos de alimentación, etc.), tipo de estudio y resultados. Extrajimos las medias (desviaciones estándar) y frecuencias (proporciones) de los resultados principales (calidad de vida física y mental) y secundarios (edad, prevalencia de AE, y horas de entrenamiento físico) para el grupo de adicción al ejercicio y para los grupos de comparación. Los revisores (JS/ $\mathrm{NE}$ ) no fueron cegados por autor o institución en ninguna etapa del proceso de selección o de recopilación de datos.

\section{Ítems de datos}

El resultado principal fue la puntuación en riesgo de adicción al ejercicio (puntuación RAE). Se ha descrito como un patrón comportamental mórbido en el cual el individuo que hace ejercicio de forma habitual pierde el control sobre sus hábitos y actúa de forma compulsiva, muestra dependencia y experimenta resultados negativos sobre su salud y su vida profesional y social (Szabo et al., 2015). La prevalencia de AE fue definida como la proporción de los participantes que obtuvieron una puntuación mayor de 24 en el EAI. Edad (años) fue definida como la duración temporal de existencia de algo. Calidad de vida (puntuación SF36) fue definida como la percepción del individuo respecto de su posición en la vida en el contexto de su propio sistema cultural y de valores, y en relación a sus objetivos, expectativas, normas y preocupaciones (Saxena, Carlson, y Billington, 2001). Trastorno de alimentación (puntuación EDI-2) (Garner, 1991; Garner, Olmstead, y Polivy, 1983) fue definida como la enfermedad caracterizada por hábitos irregulares de ingesta de alimentos y excesiva preocupación o angustia respecto de su peso o forma corporal. Estos trastornos se han convertido en una patología emergente en los países desarrollados y en vías de desarrollo, y es la tercera enfermedad crónica más común entre adolescentes, después de la obesidad y el asma (Gonzalez, Kohn, y Clarke, 2007). Las horas de ejercicio semanales es una 
variable asociada con el entrenamiento físico, tal como frecuencia semanal, intensidad o años de práctica de deporte.

\section{Riesgo de sesgo en los estudios individuales}

El riesgo de sesgo fue valorado con el STROBE (Strengthening the Reporting of Observational Studies in Epidemiology) (Vandenbroucke et al., 2014). Dos autores (JS/ $\mathrm{NE}$ ) valoraron el riesgo de sesgo de forma independiente. La calificación de la calidad de los estudios se realizó según la especialización en el campo (dirigido por JM) y se guio con una herramienta de valoración de sesgo.

\section{Gestión de datos}

La media y la desviación estándar (SD) de las características físicas de los participantes, edad y puntuación EAI, nivel de estudios, estado civil, tamaño muestral, país, tipo de ejercicio, y grupos de comparación se usaron para la determinación subjetiva de la heterogeneidad metodológica del meta-análisis (JS/JMM). Analizamos los datos para los grupos con y sin RAE usando OpenMetaAnalyst versión 3.1.0, y los expresamos como la diferencia de medias estandarizadas (ajustado con la g de Hedges) y el intervalo de confianza de 95\% (IC 95\%). Si el IC 95\% incluía cero, concluimos que no hubo efectos significativos. Valoramos la heterogeneidad estadística usando la estadística $I^{2}$ para determinar el porcentaje de variabilidad en las estimaciones del efecto debido a heterogeneidad y no a error de muestreo. Calculamos las estimaciones agrupadas del efecto y los IC 95\%, como la media ponderada de la diferencia de media estandarizada estimada en los estudios individuales. Usamos un modelo de efectos aleatorios para obtener el efecto agrupado, dado que esperábamos unos niveles altos de heterogeneidad. Realizamos un análisis posterior para obtener la edad media general, y la prevalencia media general de adicción al ejercicio, para los estudios analizados.

\section{Resultados}

\section{Participantes y estudios incluidos}

La Figura 1 detalla el flujograma PRISMA (Moher et al., 2015). Este estudio se realizó usando 5 bases de datos, y tras eliminar los duplicados, el cribado se hizo con cincuenta y dos estudios. Veinte estudios de los cincuenta y dos mencionados fueron seleccionados para la revisión sistemática; siete estudios fueron excluidos. Diecisiete de estos fueron incluidos en el meta-análisis y tres de los mismos fueron excluidos porque no analizaron variables de prevalencia o de diana. La Tabla 1 muestra las características de los participantes, y la Tabla 2 muestra detalles de los estudios. Los participantes en dichos estudios nacieron en Inglaterra ( $\mathrm{n}$ $=3)$, China $(n=1)$, Francia $(n=1)$, España $(n=3)$, Estados Unidos $(n=2)$, Hungría $(n=3)$, Italia $(n=2)$ y Dinamarca $(n=4)$. Los estudios fueron publicados entre 2005-2017. El rango de edad general de los participantes en los estudios diferentes fue entre 16-40 años. El tipo de ejercicio analizado en los estudios diversos fue muy variado, e incluyó levantamiento de pesas, CrossFit, triatlón, ciclismo, baile, gimnasio, running, levantamiento de potencia, natación, fútbol, baloncesto, ejercicio regular, deporte de combate o artes marciales, equitación, tenis, rugby, esquí, y ultramaratón. Consideramos que hubo una heterogeneidad meto-

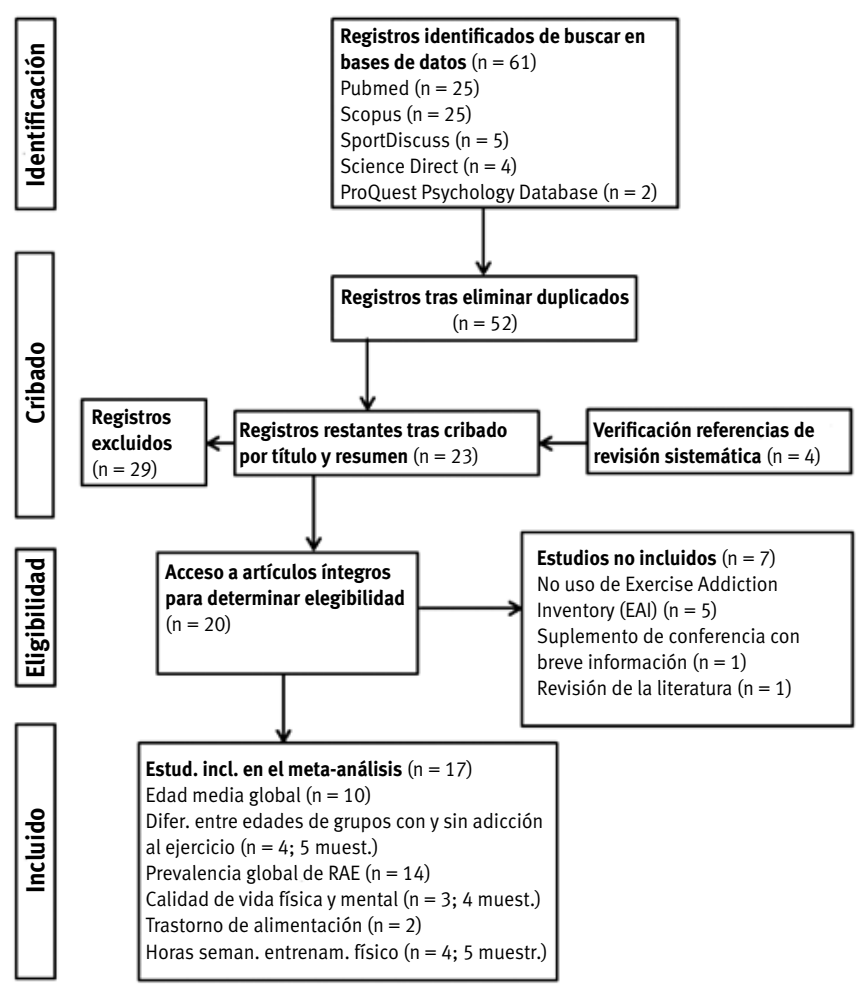

Figura 1. Flujograma PRISMA. PRISMA Preferred Reporting Items for Systematic Reviews and Meta-Analyses (Shamseer et al., 2015).

Tabla 1. Características de los participantes en los estudios incluidos

\begin{tabular}{|c|c|c|c|c|c|c|c|}
\hline Estudio & $\begin{array}{c}\text { Número } \\
\text { participantes } \\
\text { y país }\end{array}$ & $\begin{array}{l}\text { Edad (años) } \\
\text { M (SD) }\end{array}$ & $\begin{array}{c}\text { Altura (cm) } \\
\text { M (SD) }\end{array}$ & $\begin{array}{c}\text { Peso (kg) } \\
\text { M (SD) }\end{array}$ & Estudios (\%) & Estado civil (\%) & Caracterización \\
\hline Babusa et al. 2015 & $\begin{array}{c}304 \text { particip. } \\
\text { (Hungría) }\end{array}$ & $27.8 \pm 7.40$ & $179.5 \pm 6.05$ & $87.5 \pm 14.63$ & $\begin{array}{l}\text { Por debajo de estudios } \\
\text { superiores }(19 \%) \\
\text { Estudios secundarios } \\
(49,7 \%) \\
\text { Estudios universitarios } \\
(31,3 \%)\end{array}$ & $\begin{array}{l}\text { Soltero/a }(53,6 \%) \\
\text { Casado/a o con pareja } \\
(43,8 \%) \\
\text { Divorciado/a }(2,6 \%)\end{array}$ & Hombres levantadores de pesas \\
\hline Bruno et al. 2014 & $\begin{array}{l}150 \text { particip. } \\
\text { (Italia) }\end{array}$ & $\begin{array}{l}\text { Grupo de riesgo AEA } \\
\quad(29,7 \pm 7,1) \\
\text { Grupo de riesgo AEB } \\
\quad(32,2 \pm 10,1)\end{array}$ & - & - & - & - & $\begin{array}{l}\text { Gimnasia diaria } \\
\text { Grupo de riesgo AEA (hombres: } 33 \text {; } \\
\text { mujeres: 18) } \\
\text { Grupo de riesgo AEB (hombres: } 36 \text {, } \\
\text { mujeres: } 33 \text { ) }\end{array}$ \\
\hline
\end{tabular}




\begin{tabular}{|c|c|c|c|c|c|c|c|}
\hline Cunningham et al. 2016 & $\begin{array}{l}1497 \text { particip. } \\
\text { (EEUU) }\end{array}$ & $\begin{array}{l}\text { Muestra total } \\
(33,2 \pm 12,4)\end{array}$ & - & - & - & - & $\begin{array}{l}\text { Adultos ( } 608 \text { hombres, } 885 \text { mujeres, } \\
4 \text { otro) } \\
625 \text { participantes en esta muestra } \\
\text { (universitarios) con Amazon Mecha- } \\
\text { nical Turk, recibieron } \$ 1.00 \text { o } \$ 1.20 \\
\text { por completar las encuestas. } 872 \\
\text { encuestados en esta muestra (e.g., } \\
\text { triatletas, ciclistas, runners, levanta- } \\
\text { miento de potencia) mediante email, } \\
\text { medios sociales, y folletos impresos } \\
\text { para la oportunidad de ganar un } \\
\text { iPad Air. }\end{array}$ \\
\hline De la Vega et al. 2016 & 313 particip. & $\begin{array}{c}\text { Hombres }(31,1 \pm 10,1) \\
\text { Mujeres }(28,6 \pm 7,4) \\
\text { Personas que hacen } \\
\text { ejercicio sin competir, } \\
\text { como ocio }(31,5 \pm 9,3) \\
\text { Atletas locales/regiona- } \\
\text { les }(29,3 \pm 8,7) \\
\text { Atletas nacionales/ } \\
\text { internacionales } \\
(28,7 \pm 11,3) \\
\end{array}$ & - & - & - & - & $\begin{array}{l}\text { Adultos (204 hombres, } 109 \text { mujeres) } \\
\text { Los participantes practicaban } 17 \\
\text { deportes diferentes. No obstante, la } \\
\text { mayoría }(85 \%) \text { representaba siete } \\
\text { deportes, incluyendo gimnasia }(n= \\
81) \text {, atletismo }(n=73) \text {, fútbol }(n= \\
50) \text {, duatlón }(n=26) \text {, natación }(n= \\
25) \text {, triatlón }(n=24) \text {, y baloncesto } \\
(n=12) \text {. }\end{array}$ \\
\hline Griffiths et al. 2005 & $\begin{array}{l}200 \text { particip. } \\
\text { (fase 1) } \\
79 \text { particip. } \\
\text { (fase 2) } \\
\text { (Inglaterra) }\end{array}$ & $21.24 \pm 3.77$ & - & - & - & - & $\begin{array}{l}\text { Hombres y mujeres que hacen ejer- } \\
\text { cicio de forma habitual. La muestra } \\
\text { incluyó a personas que practicaban } \\
\text { muchos tipos de ejercicio diferentes. } \\
\text { El rango de edad fue } 18-40 \text { años. }\end{array}$ \\
\hline Lejoyeux et al. 2012 & $\begin{array}{l}500 \text { particip. } \\
\text { (Francia) }\end{array}$ & $\begin{array}{c}\text { Todos los participantes } \\
(29 \pm 10,3) \\
\text { DE }+(27,1 \pm 8,6) \\
\text { DE- }(29,8 \pm 10,8)\end{array}$ & - & - & $\begin{array}{l}\text { Estudios universitarios } \\
\text { en curso }(2,6 \%) \\
\text { Estudios universitarios } \\
\text { completados }(29 \%) \\
\text { Estudios secundarios } \\
\text { completados }(68 \%)\end{array}$ & - & $\begin{array}{l}\text { Clientes de una tienda de deportes } \\
\text { (para hombre y mujer) en París, con } \\
\text { edad igual o mayor de } 18 \text { años. Hom- } \\
\text { bres }(n=285) \text {; mujeres }(n=215) \text {. }\end{array}$ \\
\hline Li et al. 2015 & $\begin{array}{l}1601 \text { particip. } \\
\text { (China) }\end{array}$ & $\begin{array}{l}\text { Todos los participantes } \\
\qquad(20.51)\end{array}$ & - & - & $\begin{array}{l}\text { Estudios secundarios } \\
\text { completados }(100 \%)\end{array}$ & - & $\begin{array}{l}\text { Estudiantes de tres universidades en } \\
\text { Hunan }(C h i n a) \text {. Hombres }(n=984) ; \\
\text { mujeres }(n=617) \text {. El rango de edad } \\
\text { fue } 19-22 \text { años. }\end{array}$ \\
\hline Lichtenstein et al. 2014 & $\begin{array}{l}121 \text { particip. } \\
\text { (Dinamarca) }\end{array}$ & $\begin{array}{l}\operatorname{AEA}(28,3 \pm 7,5) \\
\operatorname{AEB}(33,5 \pm 9,1)\end{array}$ & - & - & - & - & $\begin{array}{l}\text { Hombres }(n=79) \text { y mujeres }(n= \\
\text { 42) que hacen ejercicio. El rango de } \\
\text { edad fue } 19-56 \text { años. }\end{array}$ \\
\hline Lichtenstein et al. 2015 & $\begin{array}{l}58 \text { particip. } \\
\text { (Dinamarca) }\end{array}$ & $\begin{array}{l}\operatorname{AEA}(30,3 \pm 7,9) \\
\operatorname{AEB}(37,4 \pm 6,8)\end{array}$ & - & - & - & - & Hombres aficionados al ejercicio. \\
\hline $\begin{array}{l}\text { Lichtenstein } \\
\text { y Stoving } 2016\end{array}$ & $\begin{array}{l}452 \text { particip. } \\
\text { (Dinamarca) }\end{array}$ & - & - & - & - & - & $\begin{array}{l}\text { Adolescentes y pacientes por trastor- } \\
\text { no de alimentación (rango de edad } \\
11-20 \text { años) }\end{array}$ \\
\hline $\begin{array}{l}\text { Lichtenstein } \\
\text { y Jensen } 2016\end{array}$ & $\begin{array}{l}603 \text { particip. } \\
\text { (Dinamarca) }\end{array}$ & $\begin{array}{l}\text { «3o años }(n=290) \\
\text { '31 años }(n=285)\end{array}$ & - & - & - & - & $\begin{array}{l}\text { Hombres }(n=328) \text { y mujeres }(n= \\
270) \text { que practican Crossfit. }\end{array}$ \\
\hline Maraz et al. 2015 & $\begin{array}{l}457 \text { particip. } \\
\text { (Hungría) }\end{array}$ & $32.8 \pm 8.6$ & - & - & $\begin{array}{l}\text { Estudios superiores } \\
(70 \%) \\
\text { Estudios secundarios } \\
(28 \%) \\
\text { Estudios por debajo de } \\
\text { secundarios }(2 \%)\end{array}$ & - & $\begin{array}{l}\text { Bailarines de salsa y de salón muje- } \\
\text { res }(n=305) \text { y hombres }(n=152)\end{array}$ \\
\hline Mayolas et al. 2017 & $\begin{array}{l}1577 \text { particip. } \\
\text { (España) }\end{array}$ & $\begin{array}{c}\text { AEA hombres }(37,2 \pm 8,8) \\
\text { AEB hombres }(38,4 \pm 8,4) \\
\text { AEA mujeres }(34,4 \pm 10) \\
\text { BAE mujeres }(37,4 \pm 7,4)\end{array}$ & - & - & - & - & $\begin{array}{l}\text { Ciclistas de resistencia aficionados } \\
\text { hombres }(n=751) \text { y mujeres }(n= \\
108) \text { y personas no activas hombres } \\
(n=307) \text { y mujeres }(n=411) .\end{array}$ \\
\hline Mónok et al. 2012 & $\begin{array}{l}474 \text { particip. } \\
\text { (Hungría) }\end{array}$ & $33.2 \pm 12.1$ & & & $\begin{array}{l}\text { Por debajo de estudios } \\
\text { secundarios }(18,3 \%) \\
\text { Estudios secundarios } \\
\text { completados }(35,8 \%) \\
\text { Estudios superiores o } \\
\text { universitarios }(45,9 \%)\end{array}$ & $\begin{array}{l}\text { Casado/a }(37,3 \%) \\
\text { En pareja }(10,6 \%) \\
\text { Soltero/a }(43,4 \%) \\
\text { Divorciado/a }(8,6 \%)\end{array}$ & $\begin{array}{l}\text { Hombres }(n=270) \text { y mujeres }(n= \\
\text { 204) que hacen ejercicio. }\end{array}$ \\
\hline Sicilia et al. 2013 & $\begin{array}{c}584 \text { particip. } \\
\text { (España) }\end{array}$ & $22.13 \pm 3.93$ & - & - & $\begin{array}{l}\text { Estudios superiores o } \\
\text { universitarios }(100 \%)\end{array}$ & - & $\begin{array}{l}\text { Participantes hombres (378) y mu- } \\
\text { jeres (206). } 437 \text { participantes de la } \\
\text { muestra total cursaban Ciencias de } \\
\text { la Salud y Educación Física, mientras } \\
\text { que } 147 \text { cursaban otras carreras } \\
\text { (administración de empresas, } \\
\text { psicología, etc.). El rango de edad } \\
\text { fue } 18-55 \text { años. }\end{array}$ \\
\hline Szabo y Griffiths 2007 & $\begin{array}{l}455 \text { particip. } \\
\text { (Inglaterra) }\end{array}$ & - & - & - & - & - & $\begin{array}{l}\text { Un total de } 455 \text { hombres y mujeres } \\
\text { participantes (261 estudiantes de } \\
\text { ciencias del deporte, } 194 \text { usuarios de } \\
\text { gimnasio). El rango de edad fue } 19-23 \\
\text { años para los estudiantes, y } 17-74 \\
\text { años para los usuarios. }\end{array}$ \\
\hline Szabo et al. 2013 & $\begin{array}{l}242 \text { particip. } \\
\text { (España) }\end{array}$ & $27.54 \pm 10.65$ & - & - & - & - & $\begin{array}{l}242 \text { atletas ( } 164 \text { hombres y } 78 \\
\text { mujeres) reclutados del área metro- } \\
\text { politano de Madrid. }\end{array}$ \\
\hline Villella et al. 2010 & $\begin{array}{l}2853 \text { particip. } \\
\text { (Italia) }\end{array}$ & $16.7 \pm 1.9$ & - & - & - & - & $\begin{array}{l}\text { Población de } 1142 \text { niñas ( } 40 \% \text { ) y } \\
1711 \text { niños (60\%). El rango de edad } \\
\text { fue } 13-20 \text { años. }\end{array}$ \\
\hline Warner y Griffiths 2006 & $\begin{array}{l}100 \text { particip. } \\
\text { (Inglaterra) }\end{array}$ & $37.6 \pm 12.6$ & - & - & - & - & $\begin{array}{l}\text { Usuarios de gimnasio voluntarios } \\
\text { oportunistas con edades entre } 18-74 \\
\text { años (46 hombres y } 54 \text { mujeres) }\end{array}$ \\
\hline Youngman et al. 2014 & $\begin{array}{l}1273 \text { particip. } \\
\text { (EEUU) }\end{array}$ & $37.93 \pm 9.35$ & $172.21 \pm 9.75$ & $70.23 \pm 13.20$ & $\begin{array}{l}\text { Formación profesional } \\
(\mathrm{o}, 7 \%) \\
\text { Estudios secundarios } \\
\text { completados }(1,3 \%) \\
\text { Estudiantes universita- } \\
\text { rios parciales }(5,7 \%) \\
\text { Doctorado }(14,6 \%) \\
\text { Estudios universitarios } \\
\text { completados }(41,9 \%)\end{array}$ & $\begin{array}{l}\text { Casado/a }(59,9 \%) \\
\text { Soltero/a (19,6\%) } \\
\text { Separado/Divorciado } \\
\text { (7,5\%) } \\
\text { En pareja }(12,8 \%) \\
\text { Viudo/a }(2 \%)\end{array}$ & $\begin{array}{l}\text { De los } 1273 \text { triatletas, } 589(46,3 \%) \\
\text { eran hombres y } 684(53,7 \%) \text { eran } \\
\text { mujeres. }\end{array}$ \\
\hline
\end{tabular}

Nota. M, media; SD, desviación estándar; AE, adicción al ejercicio; AEA, adicción al ejercicio alta; AEB, adicción al ejercicio baja; DE+, con dependencia del ejercicio; DE-, sin dependencia del ejercicio. 
Adicción al ejercicio medida a través del Exercise Addiction Inventory (EAl) y salud en deportistas habituales.

Una revisión sistemática y meta-análisis

Tabla 2. Detalles de los estudios incluidos que usaron el Exercise Addiction Inventory (EAI)

\begin{tabular}{|c|c|c|c|c|c|c|c|}
\hline Estudio & Tipo de ejercicio & Medidas & Grupos & $\begin{array}{l}\text { Puntuación en EAI o } \\
\text { prevalencia de AE } \\
\text { M(SE o SD) o (\%) }\end{array}$ & Variables analizadas & Estudio & Resultados \\
\hline Babusa et al. 2015 & $\begin{array}{l}\text { Levantamiento de } \\
\text { pesas }\end{array}$ & $\begin{array}{l}\text { Datos antropométricos } \\
\text { y sociodemográficos } \\
\text { Variables relacionadas } \\
\text { con el ejercicio } \\
\text { Insatisfacción con } \\
\text { el peso } \\
\text { Muscle Appearance } \\
\text { Satisfaction Scale } \\
\text { (MASS) } \\
\text { EAl }\end{array}$ & $\begin{array}{l}\text { Riesgo bajo DM ( } \mathrm{n} \\
=100) \\
\text { Riesgo moderado } \\
\text { DM }(\mathrm{n}=158) \\
\text { Riesgo alto DM ( } \mathrm{n} \\
=46)\end{array}$ & $\begin{array}{l}\text { Riesgo bajo DM } \\
(12,16 \pm 0,34) \\
\text { Riesgo moderado DM } \\
(17,42 \pm 0,31) \\
\text { Riesgo alto DM } \\
(22.70 \pm 0.53)\end{array}$ & $\begin{array}{l}\text { Dependencia de } \\
\text { Fisioculturismo } \\
\text { Comprobación } \\
\text { muscular } \\
\text { Uso de sustancias } \\
\text { Riesgo de lesión } \\
\text { Satisfacción con } \\
\text { musculatura } \\
\text { Dependencia del } \\
\text { ejercicio } \\
\text { QF de ejercicio } \\
\text { Uso de suplementos } \\
\text { Uso actual de EAA } \\
\text { Uso a lo largo de la } \\
\text { vida de EAA }\end{array}$ & Transversal & $\begin{array}{l}\text { El riesgo alto de DM obtuvo las } \\
\text { medias más altas en todas las } \\
\text { subescalas del MASS, menos } \\
\text { la subescala de satisfacción } \\
\text { con musculatura. Este grupo } \\
\text { también mostró el nivel más } \\
\text { alto de adicción al ejercicio, } \\
\text { realizó ejercicio con más } \\
\text { frecuencia, dedicó más tiempo } \\
\text { al entrenamiento físico, y } \\
\text { también mostró mayor riesgo de } \\
\text { lesión y mayor prevalencia del } \\
\text { uso de suplementos ( } 82,6 \% \text { ) } \\
\text { comparado con los otros dos } \\
\text { grupos (Riesgo bajo DM: } 49 \% \text {; } \\
\text { Riesgo moderado DM: } 70,73 \% \text { ). }\end{array}$ \\
\hline Bruno et al. 2014 & $\begin{array}{l}\text { Entrenamiento en } \\
\text { el gimnasio }\end{array}$ & $\begin{array}{l}\text { Cuestionario } \\
\text { sociodemográfico } \\
\text { EAI } \\
\text { Narcissistic } \\
\text { Personality Inventory } \\
\text { (NPI) } \\
\text { Coopersmith Self- } \\
\text { Esteem Inventory (SEI) }\end{array}$ & $\begin{array}{l}\text { Grupo de riesgo } \\
\text { AEA }(n=51) \\
\text { Grupo de riesgo } \\
\text { AEB }(n=69)\end{array}$ & $\begin{array}{l}\text { Tasa de prevalencia de } \\
\text { RAE: } 42.5 \%\end{array}$ & $\begin{array}{l}\text { Adicción al ejercicio } \\
\text { Autoestima } \\
\text { Componentes } \\
\text { narcisistas (siete } \\
\text { factores) }\end{array}$ & Transversal & $\begin{array}{l}\text { La tasa de prevalencia de } \\
\text { AE a usuarios de gimnasios } \\
\text { fue } 42,5 \% \text {; no se hallaron } \\
\text { diferencias de género en la } \\
\text { tasa de RAE encontrada. El } \\
\text { grupo de riesgo AEA informó } \\
\text { de una puntuación total en el } \\
\text { SEl significativamente más } \\
\text { bajo ( } 32,2 \text { contra } 36,4) \text { y una } \\
\text { puntuación total más alta en } \\
\text { el NPI }(20,2 \text { contra } 14,6) \text { que el } \\
\text { grupo de riesgo AEB. Narcicismo } \\
\text { y autoestima, como bloque, } \\
\text { fueron buenos predictores de } \\
\text { días semanales de ejercicio. }\end{array}$ \\
\hline Cunningham et al. 2016 & $\begin{array}{l}\text { Atletas } \\
\text { (e.g., triatletas, } \\
\text { ciclistas, runners, } \\
\text { levantadores de } \\
\text { potencia) } \\
\text { y no-atletas }\end{array}$ & $\begin{array}{l}\text { Godin Leisure- } \\
\text { Time Exercise } \\
\text { Questionnaire (GLTEQ) } \\
\text { Escala de } \\
\text { Dependencia del } \\
\text { Ejercicio-21 (EDS-21) } \\
\text { EAI } \\
\text { Compulsive Exercise } \\
\text { Test (CET) } \\
\text { Obligatory Exercise } \\
\text { Questionnaire (OEQ) } \\
\text { Commitment to } \\
\text { Exercise Scale (CES) } \\
\text { Exercise-Specific } \\
\text { Dimensional } \\
\text { Obsessive-Compulsive } \\
\text { Scale } \\
\text { Eating Disorder } \\
\text { Examination- } \\
\text { Questionnaire (EDE-0) }\end{array}$ & $\begin{array}{l}\text { Atletas } \\
(\mathrm{n}=381) \\
\text { No-atletas } \\
(\mathrm{n}=1116)\end{array}$ & $\begin{array}{l}\text { Puntuación EAl en } \\
\text { atletas }(17,4 \pm 4,93) \text { y } \\
\text { No-atletas }(15,1 \pm 5,39)\end{array}$ & $\begin{array}{l}\text { Nivel de actividad y } \\
\text { condición física } \\
\text { Dependencia del } \\
\text { ejercicio } \\
\text { Adicción al ejercicio } \\
\text { Ejercicio compulsivo } \\
\text { Ejercicio obligatorio } \\
\text { Compromiso } \\
\text { psicológico con el } \\
\text { ejercicio } \\
\text { Obsesivo } \\
\text { compulsivo } \\
\text { Trastorno de } \\
\text { alimentación }\end{array}$ & Transversal & $\begin{array}{l}\text { En este estudio, el GLTEQ } \\
\text { mostró ser inadecuado como } \\
\text { medida del ejercicio. El grupo } \\
\text { de atletas obtuvo puntuaciones } \\
\text { significativamente más altas } \\
\text { que los no-atletas en todas } \\
\text { las medidas (dependencia del } \\
\text { ejercicio, adicción al ejercicio, } \\
\text { ejercicio obligatorio, obsesivo } \\
\text { compulsivo, y trastorno de } \\
\text { alimentación), y los tamaños del } \\
\text { efecto fueron entre moderados } \\
\text { y altos. No hubo diferencias } \\
\text { en los resultados cuando se } \\
\text { ajustaron para edad y género. } \\
\text { Los coeficientes de correlación } \\
\text { entre las medidas de adicción al } \\
\text { ejercicio (EDS-21 y EAl) tuvieron } \\
\text { una correlación más significativa } \\
\text { entre sí que con las medidas de } \\
\text { ejercicio compulsivo (CES y CET). }\end{array}$ \\
\hline De la Vega et al. 2016 & $\begin{array}{l}\text { Atletas de } 17 \\
\text { deportes diferentes }\end{array}$ & $\begin{array}{l}\text { Cuestionario } \\
\text { demográfico } \\
\text { EAl } \\
\text { Escala de pasión (SPS) }\end{array}$ & $\begin{array}{l}\text { Primera } \\
\text { comparación } \\
\text { Personas que } \\
\text { hacen ejercicio } \\
\text { como ocio }(n= \\
\text { 139) vs. Atletas } \\
\text { locales/regionales } \\
\text { ( } \mathrm{n}=144 \text { ) vs. } \\
\text { Atletas nacionales/ } \\
\text { internacionales ( } \mathrm{n} \\
=30 \text { ) } \\
\text { Segunda } \\
\text { comparación } \\
\text { Atletas de deportes } \\
\text { individuales ( } \mathrm{n}= \\
244) \text { vs. Atletas de } \\
\text { deportes de equipo } \\
(\mathrm{n}=69 \text { ) }\end{array}$ & $\begin{array}{l}\text { Primera comparación } \\
\text { Personas que hacen } \\
\text { ejercicio como ocio } \\
(18 \pm 4,7) \text { vs. Atletas } \\
\text { locales/regionales } \\
(19,3 \pm 3,8) \text { vs. } \\
\text { Atletas nacionales/ } \\
\text { internacionales } \\
(20,6 \pm 3,4) \\
\text { Segunda comparación } \\
\text { Atletas de deportes } \\
\text { individuales } \\
(18,8 \pm 4,4) \text { vs. Atletas } \\
\text { de deportes de equipo } \\
(19,0 \pm 4)\end{array}$ & $\begin{array}{l}\text { Adicción al ejercicio } \\
\text { Pasión harmoniosa } \\
\text { Pasión obsesiva } \\
\text { Dedicación }\end{array}$ & Transversal & $\begin{array}{l}\text { La pasión obsesiva y la } \\
\text { dedicación al deporte } \\
\text { emergieron como predictores } \\
\text { fuertes de la adicción } \\
\text { al ejercicio. Los atletas } \\
\text { competitivos obtuvieron } \\
\text { puntuaciones más altas en } \\
\text { todas las medidas que las } \\
\text { personas que hacen ejercicio } \\
\text { sin competir. Los atletas que } \\
\text { competían a niveles bajos y } \\
\text { altos solo diferían entre sí en } \\
\text { su dedicación al deporte. Los } \\
\text { atletas de deportes de equipo } \\
\text { informaron mayor pasión } \\
\text { harmonioso y obsesivo, y } \\
\text { dedicación al deporte, pero no } \\
\text { tuvieron adicción al ejercicio } \\
\text { diferentes, comparado con los } \\
\text { atletas de deportes individuales. }\end{array}$ \\
\hline Griffiths et al. 2005 & $\begin{array}{l}\text { Personas que } \\
\text { hacen muchos } \\
\text { tipos de ejercicios } \\
\text { diferentes de } \\
\text { forma habitual } \\
\text { (deportes de } \\
\text { equipo, ejercicios } \\
\text { aeróbicos, deporte } \\
\text { de combate, } \\
\text { usuarios de } \\
\text { gimnasio, etc.) }\end{array}$ & $\begin{array}{l}\text { EAI } \\
\text { OEQ } \\
\text { EDS }\end{array}$ & Sin comparación & $\begin{array}{l}\text { Tasa de prevalencia de } \\
\text { RAE (3\%) }\end{array}$ & Adicción al ejercicio & Transversal & $\begin{array}{l}\text { Seis de los participantes ( } 3 \% \text { ) } \\
\text { obtuvieron una puntuación } \\
\text { mayor de } 24 \text { en el EAl y se } \\
\text { consideraron con RAE. También } \\
\text { se realizó una correlación } \\
\text { entre la frecuencia semanal de } \\
\text { ejercicio y las puntuaciones en } \\
\text { el EAl, y se encontró que las dos } \\
\text { variables compartían el } 29 \% \text { de } \\
\text { la varianza. }\end{array}$ \\
\hline
\end{tabular}




\begin{tabular}{|c|c|c|c|c|c|c|c|}
\hline Lejoyeux et al. 2012 & $\begin{array}{l}\text { Clientes diarios de } \\
\text { artículos deportivos } \\
\text { (tenis, natación, } \\
\text { fútbol, running, } \\
\text { fisioculturismo, } \\
\text { equitación, baile, } \\
\text { artes marciales, } \\
\text { esquí, rugby) }\end{array}$ & $\begin{array}{l}\text { Cuestionario } \\
\text { sociodemográfico } \\
\text { Tipo de ítems } \\
\text { comprados } \\
\text { EDS } \\
\text { EAI } \\
\text { DSM-IV-R } \\
\text { CAGE } \\
\text { Cuestionario } \\
\text { Fagerström } \\
\text { Índice Whiteley }\end{array}$ & $\begin{array}{l}\mathrm{DE}+(\mathrm{n}=148) \\
\mathrm{DE}-(\mathrm{n}=352)\end{array}$ & $\begin{array}{l}\text { Tasa de prevalencia de } \\
\text { RAE }(29.6 \%)\end{array}$ & $\begin{array}{l}\text { Adicción al ejercicio } \\
\text { Consumo de alcohol } \\
\text { Consumo de } \\
\text { nicotina } \\
\text { Trastornos de uso de } \\
\text { sustancias } \\
\text { Hipocondría } \\
\text { Bulimia } \\
\text { Oniomanía }\end{array}$ & Transversal & $\begin{array}{l}\text { Los autores de este estudio } \\
\text { confirman una asociación entre } \\
\text { DE y bulimia. Las personas } \\
\text { con adicción al ejercicio con } \\
\text { mayor frecuencia mostraron } \\
\text { comportamientos compulsivos } \\
\text { de ingesta de comida (binge } \\
\text { eating) y compensatorios del } \\
\text { peso. También confirmaron que } \\
\text { las personas con adicción al } \\
\text { ejercicio son hipocondríacos } \\
\text { con mayor frecuencia, } \\
\text { y que presentan un alto } \\
\text { nivel de ansiedad ante las } \\
\text { enfermedades. }\end{array}$ \\
\hline Li et al. 2015 & $\begin{array}{l}\text { Universitarios } \\
\text { miembros de clubs } \\
\text { de ejercicio físico } \\
\text { (e.g., clubes de } \\
\text { baloncesto, volibol, } \\
\text { fútbol, bádminton, } \\
\text { baile, ejercicio } \\
\text { aeróbico, artes } \\
\text { marciales, etc.) }\end{array}$ & $\begin{array}{l}\text { EAl } \\
\text { State-Trait Anxiety } \\
\text { Inventory (STAI) } \\
\text { The Center for } \\
\text { Epidemiologic Studies } \\
\text { Depression Scale } \\
\text { (CESD) } \\
\text { Well-Being Scale } \\
\text { (SWB) }\end{array}$ & $\begin{array}{l}\text { AEA }(n=181) \\
\operatorname{AEB}(n=1420)\end{array}$ & $\begin{array}{l}\text { Tasa de prevalencia de } \\
\text { RAE (11.3\%) }\end{array}$ & $\begin{array}{l}\text { Adicción al ejercicio } \\
\text { Ansiedad (ansiedad } \\
\text { estado y ansiedad } \\
\text { rasgo) } \\
\text { Depresión } \\
\text { Bienestar subjetivo }\end{array}$ & Transversal & $\begin{array}{l}\text { La adicción al ejercicio afecta } \\
\text { de forma positiva la ansiedad } \\
\text { estado, la depresión, y el } \\
\text { estado de ánimo negativo de } \\
\text { los participantes del estudio, } \\
\text { pero de forma negativa su } \\
\text { satisfacción, comportamiento } \\
\text { social y energía. }\end{array}$ \\
\hline Lichtenstein et al. 2014 & $\begin{array}{l}\text { Personas que } \\
\text { hacen ejercicio } \\
\text { reclutados de clubs } \\
\text { de fitness y equipos } \\
\text { de fútbol. También } \\
\text { universitarios } \\
\text { cursando estudios } \\
\text { de deporte y } \\
\text { medicina,y y } \\
\text { empleados de } \\
\text { comisarías de } \\
\text { policía. }\end{array}$ & $\begin{array}{l}\text { EAI } \\
\text { Self-report Short-Form } \\
\text { (SF-36) } \\
\text { Eating Disorder } \\
\text { Inventory version } 2 \\
\text { (EDI-2) } \\
\text { NEO Personality } \\
\text { Inventory Revised } \\
\text { (NEO PI-R) } \\
\text { Revised Adult } \\
\text { Attachment Scale } \\
\text { (R-AAS) }\end{array}$ & $\begin{array}{l}\text { AEA }(n=41) \\
\operatorname{AEB}(n=80)\end{array}$ & $\begin{array}{l}\text { Tasa de prevalencia de } \\
\text { RAE }(33,88 \%) \\
\text { Puntuación-EAl total } \\
(19,3 \pm 6)\end{array}$ & $\begin{array}{l}\text { Adicción al ejercicio } \\
\text { Calidad de vida } \\
\text { Síntomas de } \\
\text { trastornos de } \\
\text { alimentación } \\
\text { Rasgos de } \\
\text { personalidad } \\
\text { Estilos de apego }\end{array}$ & $\begin{array}{l}\text { Estudio de } \\
\text { caso-control }\end{array}$ & $\begin{array}{l}\text { El grupo con adicción obtuvo } \\
\text { puntuaciones más altas en } \\
\text { síntomas de trastornos de } \\
\text { alimentación, especialmente } \\
\text { en perfeccionismo, pero no tan } \\
\text { altas como las poblaciones con } \\
\text { trastornos de alimentación. El } \\
\text { grupo con adicción informó más } \\
\text { dolor corporal y lesiones. }\end{array}$ \\
\hline Lichtenstein et al. 2015 & $\begin{array}{l}\text { Aficionados que } \\
\text { hacen ejercicio } \\
\text { habitualmente, de } \\
\text { running, fitness, } \\
\text { entrenamiento con } \\
\text { pesas y ciclismo. }\end{array}$ & $\begin{array}{l}\text { EAl } \\
\text { EDI-2 } \\
\text { SF-36 } \\
\text { Muestra de sangre } \\
\text { y concentración } \\
\text { plasmática } \\
\text { Densitometría ósea } \\
\text { Estadiómetro } \\
\text { Harpenden }\end{array}$ & $\begin{array}{l}\text { AEA }(n=29) \\
\text { AEB }(n=29)\end{array}$ & $\begin{array}{l}\operatorname{AEA}(25,4 \pm 1,6) \\
\operatorname{AEB}(12,4 \pm 3,4)\end{array}$ & $\begin{array}{l}\text { Adicción al ejercicio } \\
\text { Leptin en plasma } \\
\text { Hormonas sexuales } \\
\text { Composición } \\
\text { corporal } \\
\text { Síntomas de } \\
\text { trastornos de } \\
\text { alimentación }\end{array}$ & Transversal & $\begin{array}{l}\text { Los niveles de leptin eran } \\
\text { significativamente más bajos en } \\
\text { el grupo de adicción al ejercicio } \\
\text { que en el grupo de control. } \\
\text { Los niveles de leptin eran } \\
\text { significativamente más bajos } \\
\text { en el grupo de adicción que } \\
\text { en el grupo de control, incluso } \\
\text { al ajustar por el porcentaje de } \\
\text { grasa corporal. Ninguna de las } \\
\text { personas que realizaba ejercicio } \\
\text { parecía tener un trastorno de } \\
\text { alimentación. }\end{array}$ \\
\hline $\begin{array}{l}\text { Lichtenstein y Stoving } \\
2016\end{array}$ & Sin identificar & EAI & $\begin{array}{l}\text { Adolescentes } \\
\text { en entornos } \\
\text { deportivos }(\mathrm{n}= \\
383 \text { ) } \\
\text { Pacientes de } \\
\text { una unidad de } \\
\text { trastornos de } \\
\text { alimentación ( } \mathrm{n} \\
=69 \text { ) }\end{array}$ & $\begin{array}{l}\text { Tasa de prevalencia de } \\
\text { RAE en adolescentes } \\
(5,5 \%) \\
\text { Tasa de prevalencia } \\
\text { de RAE en pacientes } \\
\text { con trastorno de } \\
\text { alimentación }(21,2 \%)\end{array}$ & $\begin{array}{l}\text { Adicción al ejercicio } \\
\text { Síntomas de } \\
\text { trastornos de } \\
\text { alimentación }\end{array}$ & Transversal & $\begin{array}{l}\text { Este estudio encontró una } \\
\text { relación positiva lineal } \\
\text { entre puntuación en el EAl y } \\
\text { "cantidad de ejercicio semanal } \\
\text { alto", "tendencia a realizar } \\
\text { ejercicio a pesar de tener una } \\
\text { lesión", "sentimientos de } \\
\text { culpabilidad cuando no se } \\
\text { hace ejercicio", "rendimiento } \\
\text { deportivo disminuido debido } \\
\text { al entrenamiento excesivo", } \\
\text { y “vida dominada por la } \\
\text { alimentación". }\end{array}$ \\
\hline $\begin{array}{l}\text { Lichtenstein y Jensen } \\
2016\end{array}$ & $\begin{array}{l}\text { Personas que } \\
\text { hacen CrossFit }\end{array}$ & $\begin{array}{l}\text { EAl } \\
\text { Cantidad de ejercicio } \\
\text { Cuatro ítems } \\
\text { adicionales }\end{array}$ & $\begin{array}{l}\text { AEA }(n=29) \\
\operatorname{AEB}(n=543)\end{array}$ & $\begin{array}{l}\text { Tasa de prevalencia de } \\
\text { RAE }(4,8 \%) \\
\text { Puntuación-EAl total } \\
(17,4 \pm 3,7)\end{array}$ & $\begin{array}{l}\text { Adicción al ejercicio } \\
\text { Ejercicio obsesivo } \\
\text { Toma de medicación } \\
\text { para hacer ejercicio } \\
\text { Hacer ejercicio a } \\
\text { pesar de dolor/ } \\
\text { lesión } \\
\text { Sentimiento de } \\
\text { culpabilidad al faltar } \\
\text { al ejercicio }\end{array}$ & Transversal & $\begin{array}{l}\text { Los autores encontraron que } \\
\text { el } 5 \% \text { de las personas que } \\
\text { practican Crossfit eran adictos } \\
\text { al ejercicio, y que los hombres } \\
\text { jóvenes tenían mayor riesgo. } \\
\text { También hallaron asociaciones } \\
\text { positivas significativas entre } \\
\text { la adicción al ejercicio y la } \\
\text { tendencia a realizar ejercicio } \\
\text { a pesar de tener una lesión, } \\
\text { sentimientos de culpabilidad } \\
\text { cuando no es posible hacer } \\
\text { ejercicio, la pasión se convierte } \\
\text { en obsesión y la toma de } \\
\text { medicación para poder hacer } \\
\text { ejercicio. }\end{array}$ \\
\hline Maraz et al. 2015 & $\begin{array}{l}\text { Bailarines de salsa } \\
\text { y bailes de salón }\end{array}$ & $\begin{array}{l}\text { Dance Addiction } \\
\text { Inventory (DAI) } \\
\text { adaptado del EAI } \\
\text { Brief Symptom } \\
\text { Inventory (BSI) } \\
\text { Mental Health } \\
\text { Continuum-short form } \\
\text { (MHC) } \\
\text { Indice de masa } \\
\text { corporal e imagen } \\
\text { corporal } \\
\text { Cuestionario SCOFF } \\
\text { McLean Screening } \\
\text { Instrument for } \\
\text { Borderline Personality } \\
\text { Disorder (MSI-BDP) } \\
\text { Dance Motivation } \\
\text { Inventory (DMI) }\end{array}$ & $\begin{array}{l}\text { Clase } 1(n=40) \\
\text { Clase } 2(n=56) \\
\text { Clase } 3(n=189) \\
\text { Clase } 4(n=111) \\
\text { Clase } 5(n=51)\end{array}$ & $\begin{array}{l}\text { Tasa de prevalencia } \\
\text { de riesgo con conflicto } \\
\text { social (11,4\%) }\end{array}$ & $\begin{array}{l}\text { Adicción a la danza } \\
\text { basada en la } \\
\text { adicción al ejercicio } \\
\text { Síntomas } \\
\text { psicológicos } \\
\text { relevantes } \\
\text { Bienestar subjetivo } \\
\text { Índice de masa } \\
\text { corporal } \\
\text { Imagen corporal } \\
\text { Síntomas de } \\
\text { trastornos de } \\
\text { alimentación } \\
\text { Síntomas de } \\
\text { trastorno límite de la } \\
\text { personalidad } \\
\text { Motivación por la } \\
\text { danza }\end{array}$ & Transversal & $\begin{array}{l}\text { Cinco clases latentes fueron } \\
\text { exploradas en base a síntomas } \\
\text { de adicción, con el } 11 \% \text { de } \\
\text { los participantes en la clase } \\
\text { más problemáática. El DAl tuvo } \\
\text { una asociación positiva con } \\
\text { angustia psiquiátrica, trastorno } \\
\text { límite de personalidad, y } \\
\text { síntomas de trastornos de } \\
\text { alimentación. La adicción a la } \\
\text { danza, valorada con el DAl, se } \\
\text { asoció con indicadores de una } \\
\text { psicopatología leve. }\end{array}$ \\
\hline
\end{tabular}


Adicción al ejercicio medida a través del Exercise Addiction Inventory (EAl) y salud en deportistas habituales. Una revisión sistemática y meta-análisis

(Continuación Tabla 2)

\begin{tabular}{|c|c|c|c|c|c|c|c|}
\hline Estudio & Tipo de ejercicio & Medidas & Grupos & $\begin{array}{l}\text { Puntuación en EAl o } \\
\text { prevalencia de AE } \\
\text { M(SE o SD) o (\%) }\end{array}$ & Variables analizadas & Estudio & Resultados \\
\hline Mayolas et al. 2017 & $\begin{array}{l}\text { Ciclistas de } \\
\text { resistencia }\end{array}$ & $\begin{array}{l}\text { Cuestionario } \\
\text { sociodemográfico } \\
\text { Short Form Survey } \\
\text { version 2.0 (SF-12V2) } \\
\text { Pittsburgh Sleep } \\
\text { Quality Index (PSQI) } \\
\text { Hospital Anxiety and } \\
\text { Depression Scale } \\
\text { (HADS) } \\
\text { International Physical } \\
\text { Activity Questionnaire } \\
\text { (IPAQ) } \\
\text { International Fitness } \\
\text { Scale (IFIS) } \\
\text { Mediterranean Diet } \\
\text { Adherence Screener } \\
\text { (MEDAS) } \\
\text { Test de Fagerström } \\
\text { de dependencia de la } \\
\text { nicotina } \\
\text { Unidades de bebida } \\
\text { estándar }\end{array}$ & $\begin{array}{l}\text { AEA hombres } \\
(n=125) \\
\text { AEB hombres } \\
(n=626) \\
\text { AEA mujeres } \\
(n=17) \\
\text { AEB mujeres } \\
(n=91) \\
\text { Hombres inactivos } \\
(n=307) \\
\text { Mujeres } \\
\text { inactivas } \\
(n=411)\end{array}$ & $\begin{array}{l}\text { Puntuación EAI AEA } \\
\text { hombres }(25,9 \pm 1,6) \\
\text { Puntuación EAI AEB } \\
\text { hombres }(17,9 \pm 3,4) \\
\text { Puntuación EAI AEA } \\
\text { mujeres }(25,2 \pm 1,3) \\
\text { Puntuación EAI AEB } \\
\text { mujeres }(17 \pm 4,1) \\
\text { Tasa de prevalencia } \\
\text { de RAE en hombres } \\
(16,64 \%) \\
\text { Tasa de prevalencia } \\
\text { de RAE en mujeres } \\
(15,74 \%)\end{array}$ & $\begin{array}{l}\text { Adicción al ejercicio } \\
\text { Calidad de vida } \\
\text { física } \\
\text { Calidad de vida } \\
\text { mental } \\
\text { Sueño } \\
\text { Ansiedad } \\
\text { Depresión } \\
\text { Riesgo cardio } \\
\text { metabólico }\end{array}$ & Transversal & $\begin{array}{l}\text { En los hombres, comparado con } \\
\text { el grupo de control, los grupos } \\
\text { de AEA y AEB tuvieron mejores } \\
\text { indicadores de calidad de vida } \\
\text { física, calidad de vida mental, } \\
\text { ansiedad y de riesgo cardio } \\
\text { metabólico. Los resultados } \\
\text { fueron similares para las } \\
\text { mujeres. Para ambos sexos, el } \\
\text { grupo de control y el grupo AEA } \\
\text { tuvieron valores comparables } \\
\text { de calidad de sueño. En los } \\
\text { hombres, comparado con el } \\
\text { grupo de control, el grupo } \\
\text { AEA tuvo peores índices de } \\
\text { depresión; no se observaron } \\
\text { diferencias entre los grupos en } \\
\text { la muestra de mujeres. No hubo } \\
\text { variaciones significativas en los } \\
\text { resultados en función de tipo } \\
\text { de ciclismo: de carretera o de } \\
\text { montaña. }\end{array}$ \\
\hline Mónok et al. 2012 & Sin identificar & $\begin{array}{l}\text { Datos demográficos } \\
\text { Preguntas sobre } \\
\text { deporte, actividad } \\
\text { física y frecuencia } \\
\text { EAI } \\
\text { EDS }\end{array}$ & $\begin{array}{l}\text { Asintomático } \\
\text { Sintomático-no } \\
\text { dependiente } \\
\text { En riesgo } \\
\text { Comparación entre } \\
\text { población que } \\
\text { hace ejercicio y } \\
\text { población general }\end{array}$ & $\begin{array}{l}\text { Tasa de prevalencia } \\
\text { de RAE en personas } \\
\text { que hacen ejercicio } \\
\text { basado en EDS }(1,9 \%) \\
\text { y EAI }(3,2 \%) \\
\text { Tasa de prevalencia } \\
\text { de RAE en población } \\
\text { general basado en EDS } \\
(0,3 \%) \text { y EAI }(0,5 \%)\end{array}$ & $\begin{array}{l}\text { Adicción al ejercicio } \\
\text { Dependencia del } \\
\text { ejercicio }\end{array}$ & Transversal & $\begin{array}{l}\text { En este estudio, el Análisis } \\
\text { Factorial Confirmatorio (AFC) } \\
\text { indica buen ajuste en ambos } \\
\text { casos de EAI y EDS y confirma } \\
\text { la estructura factorial de ambas } \\
\text { escalas. La correlación entre } \\
\text { ambas medidas fue alta. Los } \\
\text { resultados mostraron que el } \\
6,2 \% \text { (EDS) y el 10,1\% (EAI) de la } \\
\text { población se caracterizaba como } \\
\text { personas que hacen ejercicio } \\
\text { sintomático-no dependientes, } \\
\text { mientras que la proporción de } \\
\text { personas que hacen ejercicio } \\
\text { en riesgo fue de } 0,3 \% \text { y } 0,5 \% \text {, } \\
\text { respectivamente. }\end{array}$ \\
\hline Sicilia et al. 2013 & Sin identificar & $\begin{array}{l}\text { Datos demográficos } \\
\text { EAl } \\
\text { Preguntas sobre } \\
\text { frecuencia e } \\
\text { intensidad de ejercicio } \\
\text { físico }\end{array}$ & $\begin{array}{l}\text { AEA }(n=87) \\
\text { AEB }(n=440) \\
\text { Asintomático } \\
(n=57)\end{array}$ & $\begin{array}{l}\text { Tasa de prevalencia de } \\
\text { RAE }(14.9 \%)\end{array}$ & Adicción al ejercicio & Transversal & $\begin{array}{l}\text { Los resultados apoyaron la } \\
\text { estructura factorial del modelo } \\
\text { EAl. La estructura del modelo } \\
\text { no variaba por género. Un grupo } \\
\text { de } 87 \text { estudiantes (14,9\%) } \\
\text { obtuvo una puntuación total } \\
\text { igual o maayor a } 24 \text { en el EAl, } \\
\text { y se clasificó como en riesgo } \\
\text { de adicción al ejercicio. Los } \\
\text { estudiantes que hicieron } \\
\text { ejercicio más de tres días por } \\
\text { semana en niveles de intensidad } \\
\text { alto obtuvieron puntuaciones } \\
\text { más altas en adicción al } \\
\text { ejercicio que los estudiantes } \\
\text { que hicieron ejercicio en niveles } \\
\text { de intensidad bajo-medio, } \\
\text { independientemente de la } \\
\text { frecuencia. }\end{array}$ \\
\hline Szabo y Griffiths 2007 & $\begin{array}{l}\text { Estudiantes de } \\
\text { ciencias del } \\
\text { deporte y usuarios } \\
\text { de gimnasio }\end{array}$ & $\begin{array}{l}\text { Datos demográficos } \\
\text { EAI }\end{array}$ & - & $\begin{array}{l}\text { Tasa de prevalencia } \\
\text { de RAE en estudiantes } \\
\text { de ciencias del } \\
\text { deporte }(6,9 \% \text { y en } \\
\text { la población general } \\
(3,6 \%) \\
\text { Puntuación EAI } \\
\text { de estudiantes de } \\
\text { ciencias del deporte } \\
(18,6 \pm 3,8) \\
\text { Puntuación EAI de } \\
\text { la población general } \\
(17,1 \pm 3,8)\end{array}$ & Adicción al ejercicio & Transversal & $\begin{array}{l}\text { Los estudiantes de ciencias } \\
\text { del deporte obtuvieron } \\
\text { puntuaciones más altas en } \\
\text { el EAl que las personas que } \\
\text { hacen ejercicio de la población } \\
\text { general. También se encontró } \\
\text { que el } 6,9 \% \text { de los estudiantes } \\
\text { de ciencias del deporte } \\
\text { posiblemente tenían adicción } \\
\text { al ejercicio, comparado con el } \\
\text { apenas } 3,6 \% \text { de la población } \\
\text { general que hace ejercicio, } \\
\text { pero esta diferencia no llegó a } \\
\text { una significación estadística } \\
\text { conservadora. }\end{array}$ \\
\hline Szabo et al. 2013 & $\begin{array}{l}\text { Atletas } \\
\text { universitarios } \\
\text { participantes } \\
\text { en deportes } \\
\text { individuales y de } \\
\text { equipo } \\
\text { Ultramaratonistas } \\
\text { de élite }\end{array}$ & $\begin{array}{l}\text { Datos demográficos } \\
\text { EAl } \\
\text { Preguntas sobre } \\
\text { frecuencia e } \\
\text { intensidad de ejercicio } \\
\text { físico }\end{array}$ & $\begin{array}{l}\text { Atletas } \\
\text { universitarios no } \\
\text { cursando estudios } \\
\text { de deporte }(n=90) \\
\text { Atletas } \\
\text { universitarios } \\
\text { cursando ciencias } \\
\text { del deporte }(n=57) \\
\text { Ultramaratonistas } \\
\text { de élite }(n=95)\end{array}$ & $\begin{array}{l}\text { Tasa de prevalencia } \\
\text { de RAE en la muestra } \\
\text { íntegra }(12 \%) \\
\text { Puntuación EAl en } \\
\text { hombres }(19,4 \pm 3,5) \\
\text { Puntuación EAl en } \\
\text { mujeres }(18,23 \pm 3,73) \\
\text { Puntuación EAl en } \\
\text { runners de élite } \\
\text { (20,08 } \pm 3,7) \\
\text { Puntuación EAl en } \\
\text { atletas universitarios } \\
(18,41 \pm 3 ., 46 \\
\text { y } 18,23 \pm 3,34 \\
\text { respectivamente) }\end{array}$ & $\begin{array}{l}\text { Adicción al ejercicio } \\
\text { Temas subyacentes } \\
\text { del comportamiento } \\
\text { respecto al ejercicio }\end{array}$ & Transversal & $\begin{array}{l}\text { En el estudio actual, el } 7 \% \text { de } \\
\text { los atletas que estudian ciencias } \\
\text { del deporte ( } 8,8 \% \text { de todos los } \\
\text { atletas universitarios) y el } 17 \% \\
\text { de los runners de élite obtuvier } \\
\text { on puntuaciones de } 24 \text { o más } \\
\text { en el EAl. De la muestra întegra, } \\
\text { el } 12 \% \text { de los atletas obtuvo } \\
\text { puntuaciones de } 24 \text { o más en el } \\
\text { EAl. La proporción de hombres } \\
\text { fue mayor que la de mujeres } \\
\text { (21 vs. 8), con significancia } \\
\text { estadística. }\end{array}$ \\
\hline Villella et al. 2010 & Estudiantes & $\begin{array}{l}\text { Datos demográficos } \\
\text { EAl } \\
\text { South Oaks Gambling } \\
\text { Screen-Revised } \\
\text { Adolescent (SOGS-RA) } \\
\text { Compulsive Buying } \\
\text { Scale (CBS) } \\
\text { Work Addiction Risk } \\
\text { Test (WART) } \\
\text { Internet Addiction } \\
\text { Test (IAT) }\end{array}$ & - & $\begin{array}{l}\text { Tasa de prevalencia de } \\
\text { RAE }(8,5 \%) \\
\text { Tasa de prevalencia } \\
\text { de RAE en hombres } \\
(10,1 \%) \\
\text { Tasa de prevalencia } \\
\text { de RAE en mujeres } \\
(6,3 \%)\end{array}$ & $\begin{array}{l}\text { Adicción al ejercicio } \\
\text { Juego patológico } \\
\text { Oniomanía } \\
\text { Adicción al trabajo } \\
\text { Adicción a Internet }\end{array}$ & Transversal & $\begin{array}{l}\text { La prevalencia general fue del } \\
7 \% \text { para JP, } 11,3 \% \text { para ON, }, 1,2 \% \\
\text { para Al, } 7.6 \% \text { para AT, } 8,5 \% \text { para } \\
\text { AE. JP y AE fueron más común } \\
\text { entre niños, mientras que no } \\
\text { hubo efecto de género en las } \\
\text { demás condiciones. ON fue más } \\
\text { común entre los estudiantes } \\
\text { más jóvenes ( } 18 \text { años). Las } \\
\text { puntuaciones de todas estas } \\
\text { escalas tenían una correlación } \\
\text { significativa. }\end{array}$ \\
\hline
\end{tabular}




\begin{tabular}{|c|c|c|c|c|c|c|c|}
\hline Warner y Griffiths 2006 & $\begin{array}{l}\text { Entrenamiento en } \\
\text { el gimnasio }\end{array}$ & $\begin{array}{l}\text { Datos demográficos } \\
\text { EAl } \\
\text { Preguntas de } \\
\text { respuesta abierta } \\
\text { diseñadas para } \\
\text { explorar los temas } \\
\text { subyacentes del } \\
\text { comportamiento } \\
\text { respecto al ejercicio }\end{array}$ & - & $\begin{array}{l}\text { Tasa de prevalencia de } \\
\text { RAE }(8 \%) \\
\text { Puntuaciones en EAI } \\
(17,6 \pm 3,9)\end{array}$ & Adicción al ejercicio & Transversal & $\begin{array}{l}\text { Los resultados mostraron que } \\
\text { el } 8 \% \text { de los participantes } \\
\text { obtuvieron puntuaciones } \\
\text { mayores de } 24 \text { (sobre } 30 \text { y y } \\
\text { fueron definidos, en términos } \\
\text { operativos, como adictos } \\
\text { al ejercicio. Este estudio se } \\
\text { enfocó en incluir preguntas } \\
\text { abiertas cualitativas para } \\
\text { explorar las experiencias } \\
\text { positivas y negativas respecto } \\
\text { del ejercicio. Los participantes } \\
\text { comprometidos contestaron } \\
\text { con sus motivos psicológicos } \\
\text { funcionales y claros que } \\
\text { subyacen sus comportamientos } \\
\text { respecto del ejercicio. Aquellos } \\
\text { identificados como en riesgo de } \\
\text { AE indicaron que las dificultades } \\
\text { en otras áreas de sus vidas les } \\
\text { impulsaban a realizar niveles } \\
\text { alto de ejercicio potencialmente } \\
\text { nocivo. }\end{array}$ \\
\hline Youngman et al. 2014 & $\begin{array}{l}\text { Triatletas (sprint, } \\
\text { olímpico, medio } \\
\text { Ironman y Ironman) }\end{array}$ & $\begin{array}{l}\text { Datos demográficos } \\
\text { EAl } \\
\text { İtems añadidos para } \\
\text { recopilar información } \\
\text { más robusta }\end{array}$ & $\begin{array}{l}\text { AEA }(n=237) \\
\text { AEB }(n=943) \\
\text { Asintomático }(n \\
=10)\end{array}$ & $\begin{array}{l}\text { Tasa de prevalencia de } \\
\text { RAE (20\%) } \\
\text { Puntuaciones en EAI } \\
(20,82 \pm 3,32)\end{array}$ & Adicción al ejercicio & Transversal & $\begin{array}{l}\text { Los resultados indican } \\
\text { que aproximadamente el } \\
20 \% \text { de triatletas están en } \\
\text { riesgo de AE. El } 79 \% \text { está } \\
\text { comprometidos al ejercicio y } \\
\text { muestran algunos síntomas de } \\
\text { adicción al ejercicio, y el } 1 \% \text { es } \\
\text { asintomático. Los resultados } \\
\text { también muestran que las } \\
\text { mujeres triatletas tienen mayor } \\
\text { riesgo de AE que los hombres } \\
\text { triatletas. El rango de edad fue } \\
18-70 \text { años. }\end{array}$ \\
\hline
\end{tabular}

Nota. n, número de sujetos; SE, error estándar; SD, desviación estándar; MD, distrofia muscular; EAA, esteroides anabolizantes androgénicos ; AE, adicción al ejercicio; AEA, adicción al ejercicio alta; AEB, adicción al ejercicio baja; CAGE, reducir, molestado, culpable, nada más despertar; Clase 1, riesgo bajo ; Clase 2, riesgo medio sin conflictos sociales; Clase 3, riesgo medio con conflictos sociales; Clase 4, en riesgo sin conflictos sociales; Clase 5 , en riesgo con conflictos sociales; QoL, calidad de vida; JP, juego patológico; ON, Oniomanía ; Al, adicción a Internet; AT, adicción al trabajo.

Tabla 3. Riesgo de valoración de sesgo

\begin{tabular}{|c|c|c|c|c|c|c|c|c|c|c|c|c|c|c|c|c|c|c|c|c|c|c|}
\hline Estudio & $\begin{array}{c}\text { Îtem } \\
1\end{array}$ & $\begin{array}{c}\text { Îtem } \\
\mathbf{2}\end{array}$ & $\begin{array}{c}\text { Îtem } \\
\mathbf{3}\end{array}$ & $\begin{array}{c}\text { Îtem } \\
4\end{array}$ & $\begin{array}{c}\text { Îtem } \\
5\end{array}$ & $\begin{array}{c}\text { Îtem } \\
6\end{array}$ & $\begin{array}{c}\text { Îtem } \\
7\end{array}$ & $\begin{array}{c}\text { Îtem } \\
\mathbf{8}\end{array}$ & $\begin{array}{c}\text { Îtem } \\
9\end{array}$ & $\begin{array}{c}\text { İtem } \\
10\end{array}$ & $\begin{array}{c}\text { İtem } \\
\mathbf{1 1}\end{array}$ & $\begin{array}{c}\text { İtem } \\
12\end{array}$ & $\begin{array}{c}\text { Îtem } \\
13\end{array}$ & $\begin{array}{c}\text { Îtem } \\
14\end{array}$ & $\begin{array}{c}\text { Îtem } \\
15\end{array}$ & $\begin{array}{c}\text { Îtem } \\
16\end{array}$ & $\begin{array}{l}\text { Î́tem } \\
17\end{array}$ & $\begin{array}{c}\text { İtem } \\
18\end{array}$ & $\begin{array}{c}\text { Îtem } \\
19\end{array}$ & $\begin{array}{c}\text { Îtem } \\
\mathbf{2 0}\end{array}$ & $\begin{array}{c}\text { Îtem } \\
\mathbf{2 1}\end{array}$ & $\begin{array}{c}\text { İtem } \\
22 \\
\end{array}$ \\
\hline Babusa et al. 2015 & * & * & * & * & $?$ & * & * & * & * & * & * & * & $?$ & $?$ & * & * & * & * & * & * & * & $x$ \\
\hline Bruno et al. 2014 & $?$ & * & * & * & $?$ & * & * & * & $?$ & * & * & * & ? & ? & * & $?$ & * & * & * & * & $x$ & * \\
\hline Cunningham et al. 2016 & $?$ & * & * & * & * & * & * & * & * & * & * & * & * & * & * & * & * & * & * & * & $?$ & * \\
\hline De la Vega et al. 2016 & * & * & * & * & $?$ & * & * & * & * & * & * & * & $?$ & $?$ & * & $?$ & * & * & * & * & * & * \\
\hline Griffiths et al. 2005 & $?$ & * & * & * & $x$ & $?$ & $?$ & * & $x$ & * & * & $x$ & $?$ & $?$ & * & * & $?$ & * & $x$ & * & * & * \\
\hline Lejoyeux et al. 2012 & $?$ & * & * & * & * & * & * & * & $?$ & * & * & * & * & * & * & * & * & * & $?$ & * & * & $\mathrm{x}$ \\
\hline Li et al. 2015 & $?$ & * & * & * & * & * & * & * & $?$ & * & * & * & * & * & * & * & * & * & $x$ & * & * & * \\
\hline Lichtenstein et al. 2014 & * & * & * & * & * & * & * & * & $?$ & * & * & * & $?$ & $?$ & * & * & * & * & * & * & * & * \\
\hline Lichtenstein et al. 2015 & * & * & * & * & * & * & * & * & * & * & * & * & $?$ & $?$ & * & * & * & * & * & * & * & $?$ \\
\hline Lichtenstein y Stoving 2016 & $?$ & $?$ & * & $?$ & $x$ & $?$ & $?$ & $?$ & $x$ & $?$ & $x$ & $x$ & $x$ & $x$ & $?$ & $?$ & $x$ & $x$ & $x$ & $x$ & * & $?$ \\
\hline Lichtenstein y Jensen 2016 & ? & * & * & * & * & * & * & * & $?$ & * & * & $?$ & $?$ & * & * & * & * & * & * & * & * & ? \\
\hline Maraz et al. 2015 & $?$ & * & * & * & * & * & * & * & $?$ & * & * & * & $?$ & $?$ & * & * & * & * & * & * & * & $?$ \\
\hline Mayolas et al. 2017 & $?$ & * & * & * & * & * & * & * & * & * & * & * & $?$ & $?$ & * & * & * & * & * & * & * & * \\
\hline Sicilia et al. 2013 & * & * & * & * & $?$ & * & * & * & $?$ & * & * & * & $?$ & $?$ & * & * & * & * & * & * & * & $\mathrm{x}$ \\
\hline Szabo y Griffiths 2007 & * & * & * & * & $?$ & * & $x$ & $?$ & * & * & $x$ & $?$ & $x$ & $x$ & * & * & * & * & * & * & * & $?$ \\
\hline Szabo et al. 2013 & $?$ & * & * & * & $?$ & * & $?$ & * & $?$ & * & $?$ & $x$ & $x$ & $x$ & * & * & * & * & $?$ & * & * & * \\
\hline Villella et al. 2010 & * & * & * & * & * & * & * & * & $?$ & * & * & * & * & * & * & * & * & * & $?$ & $?$ & * & $?$ \\
\hline Warner y Griffiths 2006 & * & * & * & * & * & * & * & * & $x$ & * & $?$ & $?$ & $?$ & $?$ & * & * & $x$ & * & * & * & * & $x$ \\
\hline Youngman et al. 2014 & * & * & * & * & * & * & * & * & * & * & * & $?$ & * & * & * & * & * & * & * & * & * & $x$ \\
\hline
\end{tabular}

Nota. * Cumplimiento de criterios; ? cumplimiento incompleto de criterios; x No cumplimiento de criterios; Ítem 1, Título y resumen (Introducción); 2, Contexto/ fundamentos; 3, Objetivos (Métodos); 4, diseño del estudio; 5, entorno; 6, participantes; 7, Variables; 8, fuentes de datos/medidas; 9 , sesgo; 10, tamaño de estudio; 11, variables cuantitativos; 12, métodos estadísticos (resultados); 13, participantes; 14, datos descriptivos; 15, datos de los resultados; 16, resultados principales; 17, otros análisis (discusión); 18, resultados clave; 19, limitaciones; 20, interpretación; 21, generalizabilidad (otra información); 22, Financiación.

dológica amplia entre estudios respecto de las características de los participantes, tipo de ejercicio y los variables estudiados; por tanto, realizamos un meta-análisis sobre los resultados valorados usando modelos de análisis de efectos aleatorios. Pocos estudios informaron el peso, la altura, el nivel de estudios o el estado civil de la muestra. Todos los estudios incluidos fueron observacionales; no obstante, no todos los ítems para valorar el riesgo de sesgo fueron ade- cuados para todos los estudios seleccionados (Tabla 3). El ítem que más se repitió fue ítem 18.

\section{Calidad de vida física y mental (QoL)}

Analizamos la calidad de vida física y mental y, por tanto, incluimos tres estudios que compararon grupos con y sin RAE (según puntuación EAI). Todos los estudios usaron el 
cuestionario SF-36 para valorar calidad de vida. No hubo diferencias significativas en calidad de vida física entre grupos [g de Hedges = 0,057 (IC 95\% = -0,12 a 0,24) ] (Fig. 3). La heterogeneidad de los estudios respecto de calidad de vida física fue baja y no significativa $\left(I^{2}=13,43 \%, \mathrm{p}<, 325\right)$. Respecto de calidad de vida mental, el grupo sin RAE obtuvo mejor puntuación en comparación con el grupo con RAE [g de Hedges $=-0,58$ (IC 95\% = -0,95 a -0,21)] (Fig. 4). La heterogeneidad de los estudios respecto de calidad de vida mental fue alta y significativa $(I 2=73,1 \%, \mathrm{p}<, 011)$.

\section{Prevalencia de RAE}

Analizamos los trece estudios que incluyeron valores de prevalencia de RAE. Mayolas-Pi et al. (2017) analizaron dos muestras independientes (hombres y mujeres), y nosotros también realizamos este análisis independiente, como mencionado anteriormente, y también para el análisis global. La prevalencia media de RAE fue 13,2\% (IC 95\% = 9,9-16,5). La heterogeneidad de los estudios respecto de prevalencia fue muy alta $\left(I^{2}=96,2 \%, \mathrm{p}<, 001\right)$.

\section{Edad}

Realizamos un análisis estadístico de la edad media y los $S D$ de los diez estudios en los cuales fue incluida, y obtuvimos una edad media global de 28,62 años (IC 95\% = 23,36-33,88). La heterogeneidad de los estudios respecto de edad de los participantes fue muy alta $\left(I^{2}=99,95 \%\right.$, p $<, 001)$. También realizamos el análisis estadístico de edad para los grupos con y sin RAE; por tanto, seleccionados cuatro estudios que compararon ambos grupos y mostraron las medias y las desviaciones estándar para ambos. Los estudios repartieron las muestras según sus puntuaciones en el cuestionario EAI, y todos los participantes que obtuvieron una puntuación mayor de 24 fueron asignados al grupo con RAE, y los demás al grupo sin RAE. La edad fue menor en el grupo con RAE, comparado con el grupo sin RAE [g de Hedges = -0,42 (IC 95\% = -0,68 a -0,14) ] (Fig. 2) . La heterogeneidad de los estudios respecto de la edad de ambos grupos con y sin RAE fue moderada, pero significativa $\left(I^{2}=62,22 \%, \mathrm{p}=, 032\right)$.

\section{Trastornos de alimentación}

Realizamos un análisis estadístico de los dos estudios que usaron el Eating Disorder Inventory-2 (EDI-2) como medida de trastornos de alimentación para valorar dicho resultado en ambos grupos, con y sin RAE. El grupo con RAE obtuvo valores más altos que el grupo sin RAE [ $g$ de Hedges $=-0,97($ IC 95\% $=-0,65$ a $-1,29)]$ (Fig. 5) .

\section{Horas semanales de entrenamiento físico}

Realizamos un análisis estadístico de los cuatro estudios que evaluaron las horas semanales de entrenamiento físico para valorar dicho resultado en ambos grupos, con y sin RAE. El grupo con RAE entrenó más horas semanales que el grupo sin RAE [g de Hedges $=-1,48$ (IC 95\% = -0,20 a -2,75)] (Fig. 6). La heterogeneidad de los estudios respecto de horas semanales de entrenamiento físico fue muy alta $(I 2=97,79 \%, \mathrm{p}<, 001)$.

\section{Discusión}

El objetivo de este meta-análisis fue investigar las posibles diferencias en la salud general entre deportistas con RAE y sin RAE en términos de calidad de vida, conductas de alimentación, y las posibles relaciones con horas semanales de entrenamiento físico y edad. Los resultados mostraron diferencias entre los grupos, según edad, calidad de vida mental, trastornos de alimentación y horas semanales de entrenamiento físico. Aunque dichas estimaciones muestran las consecuencias negativas de la adicción al ejercicio en variables como calidad de vida mental y trastornos de alimentación, y también muestran mayor número de horas semanales de entrenamiento físico y menor edad en los grupos con RAE, consideramos que dichos hallazgos no son del todo claros y que más datos son necesarios para aumentar la confianza en la interpretación de dichos resultados, debido al número bajo de estudios desarrollados hasta la fecha en este campo.

La estrategia de búsqueda sistemática desarrollada identificó 20 estudios que cumplieron los criterios de inclusión, mientras que otra reevaluación intercultural previa del EAI

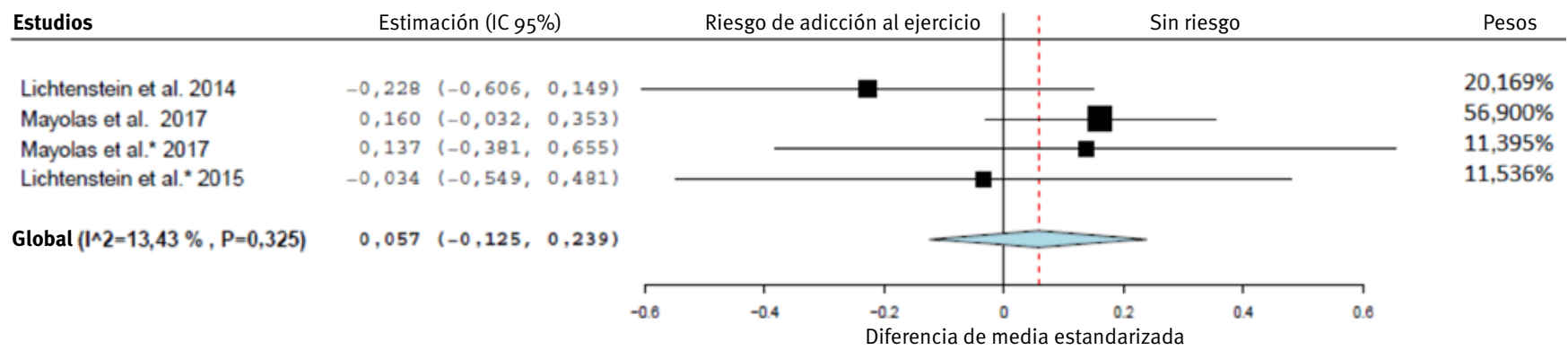

Figura 3. Estimaciones de efectos observacionales, $\mathrm{Cl} 95 \%$ y media estandarizada para calidad de vida física. IC Intervalo de confianza, diferencia de medias estandarizada. 


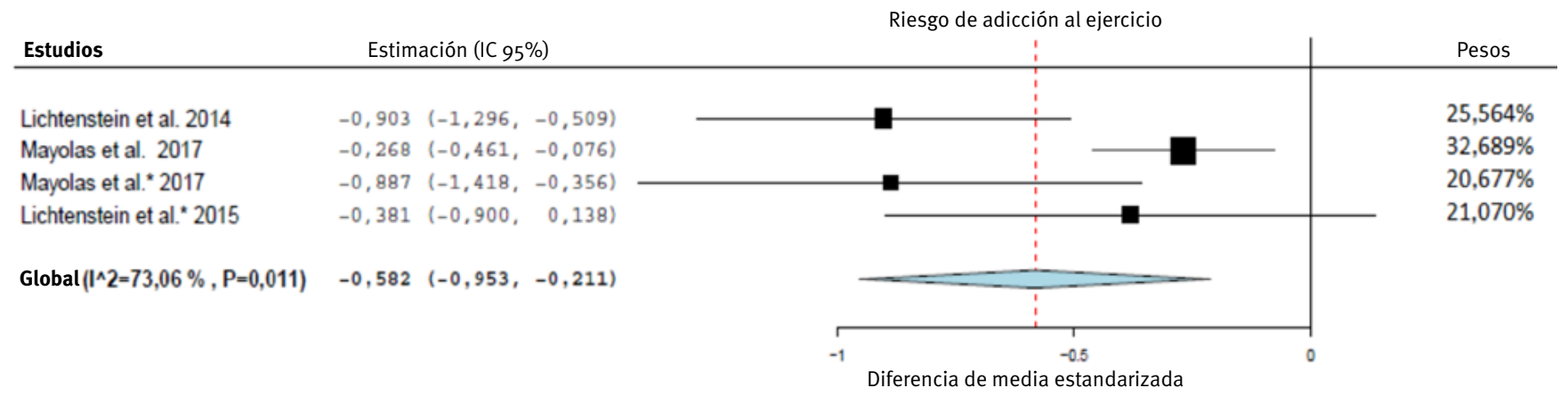

Figura 4. Estimaciones de efectos observacionales, $\mathrm{Cl} 95 \%$ y media estandarizada para calidad de vida mental. IC Intervalo de confianza, diferencia de medias estandarizada.

incluyó hasta nueve estudios (Griffiths et al., 2015). Griffiths et al. (2015) concluyeron que no eran comparables las puntuaciones en los factores del EAI de cinco países porque usaron o interpretaron la escala de maneras diferentes. No obstante, sí se puede estudiar las covariables de la adicción al ejercicio desde una perspectiva intercultural, gracias a la invarianza métrica de la escala. También salieron a la luz diferencias de género entre deportistas al interpretar la escala. Consideramos las implicaciones de los resultados, y concluimos que los resultados del estudio facilitarán un uso más robusto y fiable del EAI en estudios futuros. Otras revisiones de la literatura anteriores (Demetrovics y Kurimay, 2008; Egorov y Szabo, 2013; Landolfi, 2013; Szabo et al., 2015; Weinstein y Weinstein, 2014) que estudiaron la adicción al ejercicio incluyeron estudios diferentes que usaron diversos instrumentos de medición. El estudio actual excluyó los estudios que no usaron el EAI para evaluar RAE, o que no mostraban la prevalencia de RAE o la puntación en el EAI, para evitar la dispersión del constructo hacia otros conceptos diferentes, a pesar de estar relacionados. De todas maneras, los estudios incluidos aquí que evaluaron la prevalencia de adicción al ejercicio informaron de tasas desde el 0,3\% (Mónok et al., 2012) hasta el 42,5\% (Bruno et al., 2014). Los factores distintos (las medidas y los criterios aplicados, la selección de la muestra, el tamaño muestral y el método de muestreo) contribuyen a la amplitud del rango de tasas de adicción al ejercicio (Mónok et al., 2012). Además, esta revisión observó que la mayoría de los estudios no ofrece

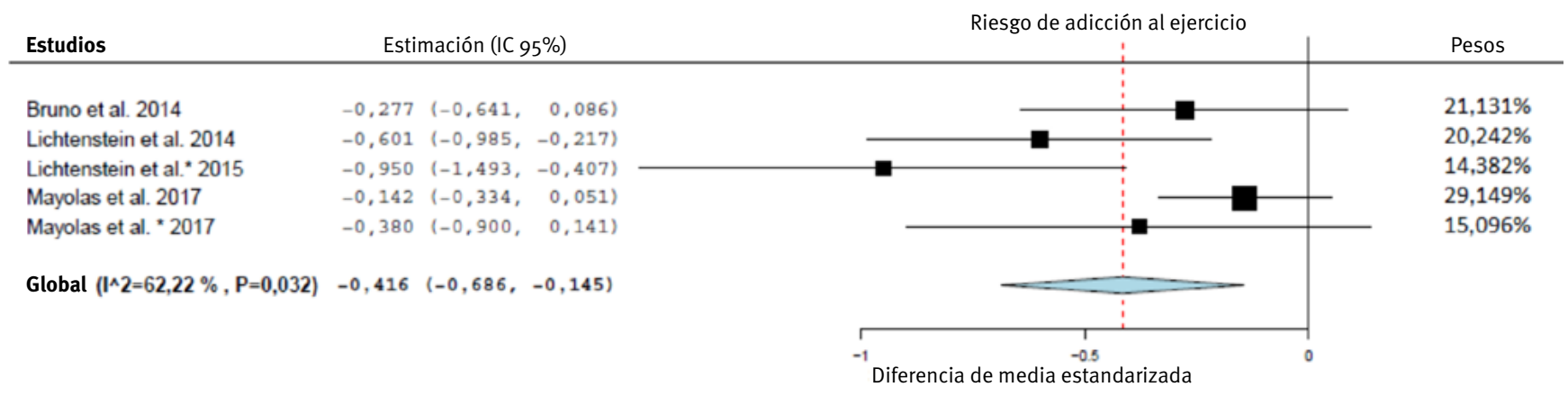

Figura 2. Estimaciones de efectos observacionales, $\mathrm{Cl} 95 \%$ y edad media estandarizada. IC Intervalo de confianza, diferencia de medias estandarizada.

\begin{tabular}{llllll} 
Estudios & Estimación (IC 95\%) & & \\
\hline
\end{tabular}

Figura 5. Estimaciones de efectos observacionales, $\mathrm{Cl} 95 \%$ y media estandarizada para trastorno de alimentación. IC Intervalo de confianza, diferencia de medias estandarizada. 
una descripción detallada de las poblaciones muestrales; por tanto, la generalización es inviable. Solo siete (Babusa, Czeglédi, Túry, Mayville y Urbán, 2015; Lejoyeux, Guillot, Chalvin, Petit y Lequen, 2012; Li et al., 2015; Maraz et al., 2015; Mónok et al., 2012; Sicilia et al., 2013; Youngman y Simpson, 2014) de los veinte estudios detalló el nivel de estudios de los participantes. Dos estudios informaron la altura y el peso (Babusa et al., 2015; Youngman y Simpson, 2014) y tres estudios informaron el estado civil (Babusa et al., 2015; Mónok et al., 2012; Youngman y Simpson, 2014). Dicha falta de información también ocurre en el estudio de Mónok et al. (Mónok et al., 2012). También hubo variedad del tipo de ejercicio, tamaño y edad de la muestra y las variables estudiadas.

\section{Prevalencia de RAE}

Como dicho anteriormente, los estudios incluidos en nuestro meta-análisis han informado tasas de prevalencia desde el 0,3\% en la población general (Mónok et al., 2012) hasta el $42,5 \%$ en usuarios de gimnasios (Bruno et al., 2014). Otros estudios informan de tasas bajas de prevalencia, menores del $5 \%$ como en el estudio desarrollado por Griffiths et al. (Griffiths et al., 2005), que informó una tasa de prevalencia del $3 \%$ en personas que hacen muchos diferentes tipos de ejercicio de manera regular, o el estudio de Lichtenstein y Jensen (Lichtenstein y Jensen, 2016), que informó una tasa de prevalencia del 4,8\% en personas que hacen CrossFit. También, el estudio de Szabo y Griffiths (Szabo y Griffiths, 2007) informó bajas tasas de prevalencia (del 6,9\% entre estudiantes de Ciencias de Educación Física y del 3,6\% en la población general que hace ejercicio). El estudio de Bruno et al. (Bruno et al., 2014) informó las tasas de prevalencia más altas, del 42,5\% entre usuarios de gimnasios. El estudio de Lichtenstein et al. (Lichtenstein et al., 2014) también informo tasas de prevalencia altas $(33,88 \%)$ en personas que hacen ejercicio en clubs de fitness, equipos de fútbol, empleados de comisarías de poli- cía, y estudiantes de medicina. El estudio de Lejoyeux et al. (Lejoyeux et al., 2012), cuya muestra estaba compuesta de clientes diarios de artículos deportivos, también informó una tasa de prevalencia alta. La prevalencia media de nuestro estudio fue del $13,2 \% \pm 3,3$. Dichos resultados sugieren que la adicción al ejercicio no es extraña, aunque algunos investigadores consideran que más bien es excepcional (Szabo, 2000; Veale, 1995). Futuros estudios con muestras homogéneas y específicas, para un tipo de ejercicio y con diseños de muestreo apropiados, sería una estrategia ideal para obtener tasas de prevalencia de adicción al ejercicio más ajustadas.

\section{Edad}

Este meta-análisis mostró que la edad era menor en el grupo con RAE, comparado con el grupo sin RAE. Esto es coherente con los resultados del estudio de De Moor et al. (De Moor, Beem, Stubbe, Boomsma, y De Geus, 2006), que observó que el ejercicio declinaba con el aumento de edad. El estudio de Szabo y Griffiths (Szabo y Griffiths, 2007) también observó que estudiantes (19-23 años), en general, también informaron más síntomas de adicción al ejercicio que la población general que hacía ejercicio (1774 años). Los resultados de Garman et al. (Garman, Hayduk, Crider, y Hodel, 2004) también mostraron una alta incidencia de adicción al ejercicio entre estudiantes que cursaban Ciencias de la Salud y Educación Física. Mejoraría la comprensión del papel del factor de edad en el RAE al ajustar la edad de las muestras, y al comparar personas que hacen ejercicio de manera habitual (con muestras más homogéneas) de diferentes edades.

\section{Calidad de vida}

A pesar de los resultados de este estudio, que el número de estudios incluidos en el meta-análisis fue bajo es una limitación importante. Varios estudios han tenido resultados positivos en cuanto a la efectividad del ejercicio como tra-

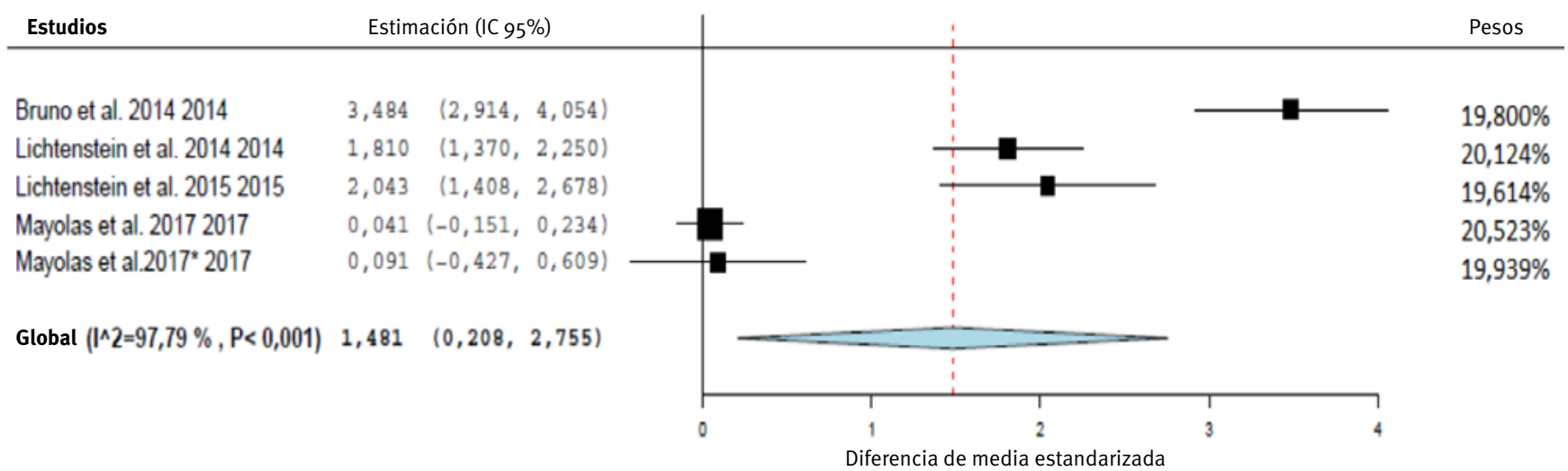

Figura 6. Estimaciones de efectos observacionales, $\mathrm{Cl} 95 \%$ y media estandarizada para horas semanales de entrenamiento físico. IC Intervalo de confianza, diferencia de medias estandarizada. 
tamiento adyuvante para los trastornos mentales (Zschucke, Gaudlitz, y Ströhle, 2013). No obstante, la adicción al ejercicio puede disminuir la calidad de vida mental de las personas, como este meta-análisis observa, aunque la escasez de estudios incluidos es una limitación considerable. Sachs y Pargman (Sachs y Pargman, 1984) también han hallado apoyo para este efecto; dicen que la adicción al ejercicio muchas veces ha sido identificada por la presencia de síntomas de abstinencia (e.g., ansiedad, depresión, sentimientos de culpabilidad e incomodidad, tensión, y excitabilidad). De todas maneras, síntomas de abstinencia son solo algunos de otros síntomas que se observan con universalidad en las adicciones comportamentales (Brown, 1993; Griffiths et al., 2005). Muchos investigadores (Griffiths, 1997; Griffiths et al., 2005; Szabo, 1995) debaten si es correcto definir la adicción al ejercicio en base a la presencia de síntomas de abstinencia, porque casi cualquier persona que hace ejercicio con regularidad informa de sentimientos psicológicos negativos cuando no puede hacer ejercicio por algún motivo inesperado (Hausenblas y Downs, 2002b; Szabo, Frenkl, y Capulo, 1997). La intensidad de dichos síntomas es el factor crítico para diferenciar entre personas que están comprometidos a hacer ejercicio habitual y personas con adicción al ejercicio (Berczik et al., 2014). Por esto, una puntuación más baja en calidad de vida mental en el grupo con RAE sugiere mayor intensidad de los síntomas psicológicos negativos. En cuanto a calidad de vida física, no observamos diferencias entre los grupos en este estudio. Estudios diferentes (Pucci, Rech, Fermino y Reis, 2012) han mostrado una asociación entre mayor cantidad de ejercicio físico y mayor puntuación en calidad de vida en el componente físico de poblaciones diferentes. No obstante, autores como Griffiths (Griffiths, 1997) sostienen que lesiones y traumatismos repetidos, causados por cantidades exageradas de ejercicio sin adecuados periodos de descanso y de recuperación, son una característica clave del grupo con adicción al ejercicio. Es más, varios autores (Chapman y De Castro, 1990; Smith, Wolfe, y Laframboise, 2001) afirman que niveles altos de ejercicio aumentan el riesgo de sufrir lesiones.

\section{Trastornos de alimentación}

Hallamos un efecto significativo respecto de los trastornos de alimentación. No obstante, el hecho que hubo pocos estudios incluidos es una limitación importante. El grupo con RAE tuvo puntuaciones más altas en trastornos de alimentación, comparado con el grupo sin RAE. De todas maneras, solo dos estudios fueron incluidos en el análisis, por lo que la generalizabilidad es muy baja. Para identificar la adicción al ejercicio, es importante distinguir entre la adicción al ejercicio -secundaria al trastorno de alimentacióny la adicción al ejercicio primaria -en la cual el ejercicio es un fin de por sí- (Lichtenstein et al., 2014). No se sabe con certeza si la adicción al ejercicio existe en la ausencia de un trastorno de alimentación. Muchas personas con adicción al ejercicio tienen un riesgo alto de desarrollar trastornos de alimentación, como anorexia nervosa y bulimia, además de un miedo al sobrepeso debido a la presión social, una imagen corporal distorsionada, la ausencia de relaciones sociales y una autoestima baja (Kreher y Schwartz, 2012; McGough, 2004). Una imagen corporal distorsionada puede, por ejemplo, llevar al uso de esteroides anabólicos androgénicos (EAA), también indicativo de un trastorno de alimentación (González-Martí, Fernández-Bustos, Jordán, y Sokolova, 2018). Además, también es característica de las personas con trastorno de alimentación la auto imposición de ejercicio obligatorio (Mondin et al., 1996).

\section{Horas semanales de entrenamiento físico}

Nuestro meta-análisis mostró diferencias en la cantidad de horas semanales de entrenamiento físico entre los grupos con y sin RAE, siendo más alta en el primero. No obstante, si consideramos la escasez de estudios incluidos en este análisis, con sus resultados diferentes, estos efectos globales no quedan claros. Por ejemplo, el estudio con el mayor tamaño muestral y, por tanto, con mayor poder estadístico (Mayolas-Pi et al., 2017), no informó de efectos significativos en este sentido. La controversia respecto del impacto de la cantidad de entrenamiento físico sobre el RAE es importante, porque algunos autores (Szabo et al., 2015) sostienen que el ejercicio excesivo no es necesariamente inadaptado ni indicativo de un trastorno. Además, estudios diferentes con atletas de resistencia son escasos, y la relación entre el RAE y la cantidad de entrenamiento físico es contradictoria (Cook et al., 2013; Szabo et al., 2013). Por otro lado, otros autores (Adkins y Keel, 2005) sugieren que el ejercicio se convierte en dañino cuando su duración, frecuencia o intensidad excede la cantidad necesaria para la salud física y, como resultado, aumenta el riesgo de sufrir una lesión física. Landolfi (Landolfi, 2013) dice que no es sorprendente que las personas adictas al ejercicio dediquen considerablemente más tiempo a la actividad física que el recomendado. De manera contraria, los atletas profesionales también dedican más horas al entrenamiento físico que la recomendada, y no muestran síntomas de adicción al ejercicio (Szabo et al., 2015). Estudios futuros que evalúen la cantidad de actividad o entrenamiento físico (frecuencia, intensidad y duración) con instrumentos como el International Physical Activity Questionnaire (IPAQ) (Craig et al., 2003) serían de ayuda para comprender mejor la influencia de la cantidad de actividad física sobre el RAE. La heterogeneidad de este meta-análisis puede deberse al tipo de deporte de los participantes, su edad, o incluso su nivel de rendimiento.

\section{Limitaciones}

Primero, un impedimento potencial de la evaluación del RAE es la amplia dispersión conceptual que resulta de 
los diferentes instrumentos de medición usados para valorarlo. Intentamos resolver este problema al seleccionar estudios que aplicaron el mismo instrumento, el EAI. No obstante, esto implicó una reducción considerable de estudios disponibles, reduciendo también las posibilidades de análisis y de generalización. Segundo, las diferencias notables entre los estudios (respecto de edad de la muestra, tipo de ejercicio, población, país, etc.) pueden explicar la alta heterogeneidad encontrada, y ha de ser objeto de estudios futuros. Tercero, no todos los estudios incluidos presentaron la información necesaria para evaluar posibles fuentes de sesgo. En este sentido, investigadores futuros deberían aportar toda la información posible sobre sus estudios para que sea de utilidad para psicólogos del deporte, entrenadores, instructores, atletas y personas que hacen ejercicio de manera habitual.

\section{Recomendaciones}

Este estudio aporta evidencia que el RAE podría estar relacionado con edad y horas semanales de entrenamiento físico y que podría afectar la calidad de vida mental y los trastornos de alimentación. No obstante, la calidad de vida física no parece ser modificada por el RAE. Con independencia de nuestros resultados, limitaciones metodológicas y conceptuales obstaculizan el desarrollo potencial del conocimiento en este campo. Más estudios son necesarios para contribuir más información, y también un punto de corte válido de la escala. Según Szabo et al. (Szabo et al., 2015), las diferencias entre los participantes (sexo, tipo de ejercicio, tamaño muestral, edad y factores sociodemográficos) impiden una interpretación correcta de los datos. Puntuaciones altas podrían reflejar actitudes diferentes hacia el ejercicio, pero no necesariamente RAE. Métodos como la entrevista personal por parte de psicólogos del deporte con personas con RAE (según los ítems del EAI, por ejemplo) podrían ser útiles para comprender la adicción al ejercicio y su conceptualización, según el caso particular. Estudios experimentales y longitudinales, mediante los cuales se pueda evaluar las diferencias en las puntuaciones entre los grupos con diferentes cantidades de entrenamiento físico y cambios en las variables relacionadas con la salud podrían dar respuestas respecto de la influencia de la cantidad de entrenamiento físico sobre el RAE. Además, estudios futuros han de priorizar el control de variables como tipo de ejercicio, edad de la muestra, comparaciones entre grupos, sexo de los participantes, variables analizados, instrumentos aplicados, etc.).

\section{Conclusiones}

El RAE parece estar relacionado con peor calidad de vida mental y con más trastornos de alimentación. Por otro lado, el RAE es más alto con menor edad de partici- pantes y más horas semanales de entrenamiento físico. La dispersión considerable de los valores de prevalencia de RAE sugiere que es necesario ser cautos al interpretar los resultados, debido a la necesidad de promover más estudios en este campo, enfocados en variables y poblaciones específicos.

\section{Conflicto de intereses}

Los autores declaran la inexistencia de conflictos de intereses respecto de este análisis.

\section{Referencias}

Adams, J. M., Miller, T. W. y Kraus, R. F. (2003). Exercise Dependence: Diagnostic and Therapeutic Issues for Patients in Psychotherapy. Journal of Contemporary Psychotherapy, 33: 93-107. doi:10.1023/A:1022883104269.

Adkins, E. C. y Keel, P. K. (2005). Does "excessive" or "compulsive" best describe exercise as a symptom of bulimia nervosa? International Journal of Eating Disorders, 38, 24 29. doi:10.1002/eat.20140.

Allegre, B., Souville, M., Therme, P. y Griffiths, M. (2009). Definitions and measures of exercise dependence. Addiction Research and Theory, 14, 631-646. doi:10.1080/16066350600903302.

American Psychiatric Association (2013). Diagnostic and statistical manual of mental disorders (5th ed.). Arlington: American Psychiatric Publishing.

Babusa, B., Czeglédi, E., Túry, F., Mayville, S. B. y Urbán, R. (2015). Differentiating the levels of risk for muscle dysmorphia among Hungarian male weightlifters: a factor mixture modeling approach. Body Image, 12, 14-21. doi:10.1016/j.bodyim.2014.09.001.

Berczik, K., Griffiths, M., Szabó, A., Kurimay, T., Kökönyei, G., Urbán, R. y Demetrovics, Z. (2014). Exercise addiction - the emergence of a new disorder. Australian Epidemiologist, 21, 36-40.

Berczik, K., Szabó, A., Griffiths, M. D., Kurimay, T., Kun, B., Urbán, R. y Demetrovics, Z. (2012). Exercise addiction: symptoms, diagnosis, epidemiology, and etiology. Substance Use y Misuse, 47, 403-417. doi:10.3109/10826084.2 011.639120.

Brown, R. (1993). Some Contributions of the Study of Gambling to the Study of Other Addictions. In Gambling Behaviour and Problem Gambling (pp. 241-272). Reno: University of Nevada Press.

Bruno, A., Quattrone, D., Scimeca, G., Cicciarelli, C., Romeo, V. M., Pandolfo, G.,... Muscatello, M. R. (2014). Unraveling exercise addiction: the role of narcissism and self-esteem. Journal of Addiction, 2014, 987841. doi:10.1155/2014/987841.

Chapman, C. L. y De Castro, J. M. (1990). Running addiction: measurement and associated psychological charac- 
teristics. The Journal of Sports Medicine and Physical Fitness, 30, 283-290.

Cook, B., Karr, T. M., Zunker, C., Mitchell, J. E., Thompson, R., Sherman, R.,... Wonderlich, S. A. (2013). Primary and secondary exercise dependence in a community-based sample of road race runners. Journal of Sport and Exercise Psychology, 35, 464-469. doi.org: /10.1123/ jsep.35.5.464.

Craig, C. L., Marshall, A. L., Sjöström, M., Bauman, A. E., Booth, M. L., Ainsworth, B. E.,... Oja, P. (2003). International physical activity questionnaire: 12-country reliability and validity. Medicine y Science in Sports y Exercise, 35, 1381-1395. doi:10.1249/01.MSS.0000078924.61453.FB.

Cunningham, H. E., Pearman, S. y Brewerton, T. D. (2016). Conceptualizing primary and secondary pathological exercise using available measures of excessive exercise. International Journal of Eating Disorders, 49, 778-792. doi:10.1002/eat.22551.

Dalle Grave, R., Calugi, S. y Marchesini, G. (2008). Compulsive exercise to control shape or weight in eating disorders: prevalence, associated features, and treatment outcome. Comprehensive Psychiatry, 49, 346-352. doi:10.1016/j.comppsych.2007.12.007.

Davis, C. (2000). Exercise abuse. International Journal of Sport Psychology, 31, 278-289.

De la Vega, R., Parastatidou, I. S., Ruiz-Barquin, R. y Szabo, A. (2016). Exercise Addiction in Athletes and Leisure Exercisers: The Moderating Role of Passion. Journal of Behavioral Addictions, 5, 325-331. doi:10.1556/2006.5.2016.043.

De Moor, M. H., Beem, A. L., Stubbe, J. H., Boomsma, D. I. y De Geus, E. J. (2006). Regular exercise, anxiety, depression and personality: a population-based study. Preventive Medicine, 42, 273-279. doi:10.1016/j.ypmed.2005.12.002.

Demetrovics, Z. y Kurimay, T. (2008). Exercise addiction: a literature review. Psychiatria Hungarica, 23, 129-141.

Egorov, A. Y. y Szabo, A. (2013). The exercise paradox: An interactional model for a clearer conceptualization of exercise addiction. Journal of Behavioral Addictions, 2, 199-208. doi:10.1556/JBA.2.2013.4.2.

Freimuth, M., Moniz, S. y Kim, S. R. (2011). Clarifying exercise addiction: differential diagnosis, co-occurring disorders, and phases of addiction. International Journal of Environmental Research and Public Health, 8, 4069-4081. doi:10.3390/ijerph8104069.

Garman, J. F., Hayduk, D. M., Crider, D. A. y Hodel, M. M. (2004). Occurrence of exercise dependence in a college-aged population. Journal of American College Health, 52, 221-228. doi:10.3200/JACH.52.5.221-228.

Garner, D. M. (1991). Eating disorder inventory-2. Professional manual. Odessa, FL: Psychological Assessment Research, Inc.
Garner, D. M., Olmstead, M. P. y Polivy, J. (1983). Development and validation of a multidimensional eating disorder inventory for anorexia nervosa and bulimia. International Journal of Eating Disorders, 2, 15-34. doi:10.1002 /1098-108X(198321) 2:2<15::AID-EAT2260020203>3.0. CO;2-6.

Gonzalez, A., Kohn, M. R. y Clarke, S. D. (2007). Eating disorders in adolescents. Australian Family Physician, 36, 614-619.

González-Martí, I., Fernández-Bustos, J. G., Jordán, O. R. C. y Sokolova, M. (2018). Dismorfia Muscular: detección del uso-abuso de esteroides anabolizantes androgénicos en una muestra española. Adicciones, 30, 243-250. doi:10.20882/adicciones.853.

Goodman,A. (1990).Addiction:definitionandimplications. Addiction, 85, 1403-1408. doi:10.1111/j.1360-0443.1990. tb01620.x.

Griffiths, M. (1996). Behavioural addiction: an issue for everybody?. Employee Counselling Today, 8, 19-25. doi:10.1108/13665629610116872.

Griffiths, M. (1997). Exercise Addiction: A Case Study. Addiction Research, 5, 161-168. doi:10.3109/16066359709005257.

Griffiths, M. D., Szabo, A. y Terry, A. (2005). The exercise addiction inventory: a quick and easy screening tool for health practitioners. British Journal of Sports Medicine, 39, e30. doi:10.1136/bjsm.2004.017020.

Griffiths, M. D., Urban, R., Demetrovics, Z., Lichtenstein, M. B., de la Vega, R., Kun, B.,... Szabo, A. (2015). A cross-cultural re-evaluation of the Exercise Addiction Inventory (EAI) in five countries. Sports Medicine Open, 1, 5. doi:10.1186/s40798-014-0005-5.

Hausenblas, H. A. y Downs, D. S. (2002a). How much is too much?. The development and validation of the exercise dependence scale. Psychology y Health, 17, 387-404. doi:1 $0.1080 / 0887044022000004894$.

Hausenblas, H. A. y Downs, D. S. (2002b). Exercise dependence: a systematic review. Psychology of Sport and Exercise, 3, 89-123. doi:http://dx.doi.org/10.1016/S14690292(00)00015-7.

Kokkinos, P. y Myers, J. (2010). Exercise and physical activity: clinical outcomes and applications. Circulation, 122, 1637-1648. doi:10.1161/CIRCULATIONAHA.110.948349.

Kreher, J. B. y Schwartz, J. B. (2012). Overtraining syndrome: a practical guide. Sports Health, 4, 128-138. doi:10.1177/1941738111434406.

Landolfi, E. (2013). Exercise addiction. Sports Medicine, 43, 111-119. doi:10.1007/s40279-012-0013-x.

Lejoyeux, M., Guillot, C., Chalvin, F., Petit, A. y Lequen, V. (2012). Exercise dependence among customers from a Parisian sport shop. Journal of Behavioral Addictions, 1, 28-34. doi:10.1556/JBA.1.2012.1.3. 
Li, M., Nie, J. y Ren, Y. (2015). Effects of exercise dependence on psychological health of Chinese college students. Psychiatria Danubina, 27(4), 413-419. ISSN: 03535053

Lichtenstein, M. y Stoving, R. K. (2016). Exercise addiction: Identification and prevalence in physically active adolescents and young eating disordered patients. European Psychiatry, 33, S116-S117. doi:10.1016/j.eurpsy.2016.01.127.

Lichtenstein, M. B., Andries, A., Hansen, S., Frystyk, J. y Stoving, R. K. (2015). Exercise addiction in men is associated with lower fat-adjusted leptin levels. Clinical Journal of Sport Medicine, 25, 138-143. doi:10.1097/ jsm.0000000000000110.

Lichtenstein, M. B., Christiansen, E., Bilenberg, N. y Stoving, R. K. (2014). Validation of the exercise addiction inventory in a Danish sport context. Scandinavian Journal of Medicine y Science in Sports, 24, 447-453. doi:10.1111/ j.1600-0838.2012.01515.x.

Lichtenstein, M. B., Christiansen, E., Elklit, A., Bilenberg, N. y Stoving, R. K. (2014). Exercise addiction: a study of eating disorder symptoms, quality of life, personality traits and attachment styles. Psychiatry Research, 215, 410416. doi:10.1016/j.psychres.2013.11.010.

Lichtenstein, M. B. y Jensen, T. T. (2016). Exercise addiction in CrossFit: Prevalence and psychometric properties of the Exercise Addiction Inventory. Addictive Behaviors Reports, 3, 33-37. doi:10.1016/j.abrep.2016.02.002.

Maraz, A., Urban, R., Griffiths, M. D. y Demetrovics, Z. (2015). An empirical investigation of dance addiction. PLoS One, 10, e0125988. doi:10.1371/journal. pone.0125988.

Mayolas-Pi, C., Simón-Grima, J., Peñarrubia-Lozano, C., Munguía-Izquierdo, D., Moliner-Urdiales, D. y Legaz-Arrese, A. (2017). Exercise addiction risk and health in male and female amateur endurance cyclists. Journal of Behavioral Addictions, 6, 74-83. doi:10.1556/2006.6.2017.018.

McGough, S. (2004). (Accesed 20 Mar 2017). Exercise addiction and eating disorders. Recuperado de http:/ /www. mclean.harvard.edu/pdf/news/fitnessmanage0704.pdf

Moher, D., Shamseer, L., Clarke, M., Ghersi, D., Liberati, A., Petticrew,... PRISMA-P Group (2015). Preferred reporting items for systematic review and meta-analysis protocols (PRISMA-P) 2015 statement: elaboration and explanation. Systematic Reviews, 4. doi:10.1186/20464053-4-1.

Mondin, G. W., Morgan, W. P., Piering, P. N., Stegner, A. J., Stotesbery, C. L., Trine, M. R. y Wu, M. Y. (1996). Psychological consequences of exercise deprivation in habitual exercisers. Medicine y Science in Sports y Exercise, 28, 1199-1203.

Mónok, K., Berczik, K., Urbán, R., Szabo, A., Griffiths, M. D., Farkas, J.,... Demetrovics, Z. (2012). Psychometric properties and concurrent validity of two exercise addiction measures: A population wide study. Psychology of Sport and Exercise, 13, 739-746. doi:10.1016/j.psychsport.2012.06.003.

Ogden, J., Veale, D. y Summers, Z. (1997). The Development and Validation of the Exercise Dependence Questionnaire. Addiction Research, 5, 343-356. doi:10.3109/16066359709004348.

Pasman, L. y Thompson, J. (1988). Body image and eating disturbance in obligatory runners, obligatory weightlifters, and sedentary individuals. International Journal of Eating Disorders, 7, 759-769. doi:10.1002/1098108X(198811).

Pucci, G., Rech , R., Fermino, R. y Reis, R. (2012). Association between physical activity and quality of life in adults. Revista de Saúde Pública, 46, 1. doi:10.1590/S003489102012000100021

Sachs, M. L. y Pargman, D. (1984). Running addiction. In M. L. Sachs y G. W. Buffone (Eds.), Running as therapy: An integrated approach (pp. 231-252): University of Nebraska Press.

Saxena, S., Carlson, D. y Billington, R. (2001). The WHO quality of life assessment instrument (WHOQOL-Bref): the importance of its items for cross-cultural research. Quality of Life Research, 10, 711-721. doi: 10.1023/A:1013867826835.

Sicilia, A., Alias-Garcia, A., Ferriz, R. y Moreno-Murcia, J. A. (2013). Spanish adaptation and validation of the Exercise Addiction Inventory (EAI). Psicothema, 25, 377-383. doi:10.7334/psicothema2013.21.

Smith, J. E., Wolfe, B. L. y Laframboise, D. E. (2001). Body image treatment for a community sample of obligatory and nonobligatory exercisers. International Journal of Eating Disorders, 30, 375-388. doi:10.1002/eat.1099.

Szabo, A. (1995). The impact of exercise deprivation on well-being of habitual exercises. Australian Journal of Science and Medicine in Sport, 27, 68-75.

Szabo, A. (2000). Physical Activity and Psychological Well-Being. In S. Biddle, K. Fox, y S. Boutcher (Eds.), Physical activity and psychological dysfunction (pp. 130-153). London: Routledge.

Szabo, A., Frenkl, R. y Caputo, A. (1997). Relationship between addiction to running, commitment to running, and deprivation from running: A study on the internet. European Yearbook of Sport Psychology, 1, 130-147.

Szabo, A. y Griffiths, M. (2007). Exercise addiction in British sport science students. International Journal of Mental Health and Addiction, 5, 25-28. doi:10.1007/s11469-0069050-8.

Szabo, A., Griffiths, M. D., de La Vega Marcos, R., Mervó, B. y Demetrovics, Z. (2015). Methodological and Conceptual Limitations in Exercise Addiction Research. Yale Journal of Biology and Medicine, 88, 303-308. 
Szabo, A., De la Vega R., L., Ruiz-Barquín, R. y Rivera, O. (2013). Exercise addiction in Spanish athletes: Investigation of the roles of gender, social context and level of involvement. Journal of Behavioral Addictions, 2, 249-252. doi:10.1556/jba.2.2013.4.9.

Taranis, L., Touyz, S. y Meyer, C. (2011). Disordered eating and exercise: development and preliminary validation of the compulsive exercise test (CET). European Eating Disorders Review, 19, 256-268. doi:10.1002/erv.1108.

Vandenbroucke, J. P., Von Elm, E., Altman, D. G., Gotzsche, P. C., Mulrow, C. D., Pocock, S. J.,... Egger, M. (2014). Strengthening the Reporting of Observational Studies in Epidemiology (STROBE): explanation and elaboration. International Journal of Surgery, 12, 15001524. doi:10.1016/j.ijsu.2014.07.014.

Veale, D. (1995). Does Primary Exercise Dependence really exist? In Exercise Addiction: Motivation for participation in sport and exercise (pp. 71-75): British Psychological Society.

Villella, C., Martinotti, G., Di Nicola, M., Cassano, M., La Torre, G., Gliubizzi, M. D.,... Conte, G. (2011). Behavioural addictions in adolescents and young adults: results from a prevalence study. Journal of Gambling Studies, 27, 203-214. doi:10.1007/s10899-010-9206-0.

Warner, R. y Griffiths, M. D. (2006). A qualitative thematic analysis of exercise addiction: An exploratory study. International Journal of Mental Health and Addiction, 4, 13-26. doi:10.1007/s11469-006-9000-5.

Weinstein, A., Maayan, G. y Weinstein, Y. (2015). A study on the relationship between compulsive exercise, depression and anxiety. Journal of Behavioral Addictions, 4, 315318. doi:10.1556/2006.4.2015.034.

Weinstein, A. y Weinstein, Y. (2014). Exercise addictiondiagnosis, bio-psychological mechanisms and treatment issues. Current Pharmaceutical Desing, 20, 4062-4069. doi: 10.2174/13816128113199990614.

Youngman, J. y Simpson, D. (2014). Risk for Exercise Addiction: A Comparison of Triathletes Training for Sprint-, Olympic-, Half-Ironman-, and Ironman-Distance Triathlons. Journal of Clinical Sport Psychology, 8, 19-37. doi:10.1123/jcsp.2014-0010.

Zschucke, E., Gaudlitz, K. y Ströhle, A. (2013). Exercise and Physical Activity in Mental Disorders: Clinical and Experimental Evidence. Journal of Preventive Medicine and Public Health, 46, S12-S21. doi:10.3961/jpmph.2013.46.S.S12. 